\title{
Chern-Simons-Witten Invariants of Lens Spaces and Torus Bundles, and the Semiclassical Approximation
}

\author{
Lisa C. Jeffrey ${ }^{\star}$ \\ Department of Pure Mathematics and Mathematical Statistics, University of Cambridge, \\ 16 Mill Lane, Cambridge CB2 1SB, UK ${ }^{\star \star}$
}

Received August 15, 1991; in revised form February 10, 1992

\begin{abstract}
We derive explicit formulas for the Chern-Simons-Witten invariants of lens spaces and torus bundles over $S^{1}$, for arbitrary values of the level $k$. Most of our results are for the group $G=S U(2)$, though some are for more general compact groups. We explicitly exhibit agreement of the limiting values of these formulas as $k \rightarrow \infty$ with the semiclassical approximation predicted by the Chern-Simons path integral.
\end{abstract}

\section{Introduction}

New invariants of 3-manifolds were introduced by Witten [40] using Chern-Simons gauge theory. In this paper we study these invariants explicitly for certain families of three-manifolds. Our ultimate objective is to verify for these families certain properties of the invariants derived heuristically using the Feynman path integral.

Witten [40] specified his three-manifold invariants in terms of the axioms of topological quantum field theory (TQFT), based on modular functors derived from conformal field theory [30]. This definition can be made rigorous, and is the one we shall use here. Three-manifold invariants were also defined combinatorially by Reshetikhin and Turaev [36], using modular Hopf algebras associated to quantum groups. Many properties of the invariants of Reshetikhin and Turaev have been established by Kirby and Melvin [23] from this point of view. It had always been expected that Reshetikhin and Turaev's definition would prove to be equivalent to the definition using the topological field theory axioms: this was, however, only proved recently by Walker ([39], Sects. 9-11).

Pure mathematicians who have worked on these invariants have focused almost exclusively on the combinatorial or TQFT definition. On the other hand, Witten and

\footnotetext{
* Partially supported by an NSF Graduate Fellowship

$\star \star$ Address as of September 1, 1991: School of Natural Science, Institute for Advanced Study, Princeton, NJ 08540; USA
} 
other physicists also use a complementary approach to the invariants, the Feynman path integral. Here, the Chern-Simons partition function is defined as follows. One takes a compact Lie group $G$ and considers the trivial principal $G$ bundle $M \times G$ over a 3-manifold $M$. One then performs the path integral over connections $A$ on $M \times G$ :

$$
Z(M, k)=\int \mathscr{D} A \exp 2 \pi i k \operatorname{CS}(A),
$$

where the Chern-Simons functional is defined by

$$
\mathrm{CS}(A)=\frac{1}{8 \pi^{2}} \int_{M} \operatorname{Tr}\left(A d A+\frac{2}{3} A^{3}\right) .
$$

The partition function depends on an integer parameter $k$, the level.

The path integral encodes certain properties of the Chern-Simons-Witten partition function that are not superficially obvious from the combinatorial definition. In particular, the path integral predicts a formula for the asymptotic behaviour of $Z(M, k)$ in the limit of large $k$. This formula $((5.1)$ below) is expressed as a sum over the flat connections $A_{i}$ on $M$ (assuming these form a discrete set); it involves the Chern-Simons invariants $\operatorname{CS}\left(A_{i}\right)$, the Reidemeister-Ray-Singer torsion $\tau\left(M, A_{i}\right)$ and the spectral flow $I_{A_{i}}$ of a certain family of operators parametrized by a path of connections from the product connection $A_{0}$ to $A_{i}$.

In the TQFT definition, on the other hand, the more natural parameter is

$$
r=k+h \text {. }
$$

The invariant, which we shall denote by $Z(M, r)$, is expressed as a polynomial in a root of unity whose order is some multiple of $r$; the number of terms in the polynomial is typically some power of $r$. Thus the asymptotic behaviour as $r \rightarrow \infty$ is far from obvious. The axioms of topological quantum field theory $[3,39]$ treat each value of $r$ separately and provide no axiomatic framework for studying the functional dependence on $r$ of the family of partition functions parametrized by $r$. From the axiomatic point of view one can only study the behaviour as $r \rightarrow \infty$ after one has obtained an explicit expression for the partition function of a given 3-manifold for all values of $r$.

Evidence for the validity of the asymptotic expansion predicted by the path integral has nonetheless been provided by Freed and Gompf [11], who investigated the $r \rightarrow \infty$ limit numerically for lens spaces and Brieskorn spheres. The interest of the asymptotic formula is not so much its effectiveness as a tool for distinguishing 3-manifolds: it is rather that the gauge-theoretic quantities involved in the asymptotic expansion do not enter in any obvious way in the combinatorial definition of the invariants. The path integral nonetheless predicts a relation between these gauge theoretic quantities and the combinatorial definition: finding this relation is a formidable challenge.

This paper has two main objectives. One is to provide explicit formulas for the Chern-Simons-Witten invariants (defined via TQFT) for certain families of threemanifolds for which an explicit treatment is possible: lens spaces and torus bundles over $S^{1}$. The second is to write these formulas in a form where the behaviour as $r \rightarrow \infty$ is obvious. In this way we explicitly exhibit the first term in the $r \rightarrow$ $\infty$ asymptotic expansion for these families of 3-manifolds. This work is the first rigorous confirmation of the validity of the asymptotic expansion for a class of 3manifolds; we hope to extend the methods presented here to treat a larger class of examples. More speculatively, we suggest that the partition functions $Z(M, r)$ for 
general three-manifolds $M$ will satisfy certain number theoretic properties that lead to the asymptotic formula. These properties remain to be discovered, but the methods presented here may give some indication of the general ingredients involved.

The lens space $L(p, q)$ is obtained by gluing two solid tori using an element of $S L(2, \mathbb{Z})$. We derive an exact formula (Theorem 3.4) for the $S U(2)$ Chern-SimonsWitten invariant $Z(L(p, q), r)$ for arbitrary $r \in \mathbb{Z}$, without assumptions that $r$ be coprime to any primes determined by $p$ and $q$. Theorem 3.7 gives the specialization of this formula to the case when $(r, p)=1$. It follows from our formula that $Z(L(p, q), r)$ distinguishes a large number of $L(p, q)$ up to orientation preserving homeomorphism, provided $p \neq 2(\bmod 4)$. (It does not, however, distinguish all lens spaces: see Remark 3.9.) We also derive the $S U(2)$ Witten invariant $Z\left(\Sigma_{U}, r\right)$ for the torus bundle $\Sigma_{U}$ over $S^{1}$ with monodromy specified by an element $U$ of $S L(2, \mathbb{Z}$ ) (formula (4.8)). For one family of torus bundles parametrized by $\mathbb{Z}$, we also derive the Witten invariant (4.16) for an arbitrary simply connected simply laced compact Lie group $G$.

For these families of three-manifolds $M$, we define $Z(M, r)$ using the representation $\mathscr{B}$ of $S L(2, \mathbb{Z})$ (the torus mapping class group) obtained from conformal field theory, on the vector space associated to the torus by the topological field theory. For lens spaces, this definition of $Z(M, r)$ is transparently equivalent to the definition one would obtain from a surgery description (see, for instance, Lemma 3.2.7 of [23].) For torus bundles, the equivalence with the combinatorial definition in terms of a surgery description is not obvious, but follows as noted above from the work of Walker [39].

The representation $\mathscr{B}$ of $S L(2, \mathbb{Z})$ is usually specified in terms of two standard generators $S, T$ of $P S L(2, \mathbb{Z})$ : we derive a formula (Proposition 2.6) expressing it explicitly in terms of the four coefficients in a $2 \times 2$ matrix with determinant 1 . This formula is obtained by an inductive argument using a classical identity from number theory, the reciprocity formula for Gauss sums, which follows from the Poisson summation formula.

In Sect. 5, we explicitly demonstrate agreement of these results with the leading order term in the path integral asymptotic expansion for $Z(M, r)$ as $r \rightarrow \infty$, which was described in [11] and [40].

The results presented in this paper are part of my Oxford University D. Phil. thesis: I would like to thank Prof. M. F. Atiyah for supervising this work. I also gratefully acknowledge helpful conversations with D. Freed, S. Garoufalidis, R. Kirby, and D. Zagier.

This paper is organized as follows. In Sect. 2, we construct an explicit formula for the representation of the torus mapping class group $S L(2, \mathbb{Z})$. Section 3 applies this formula to obtain formulas for the Chern-Simons-Witten invariants for lens spaces for $G=S U(2)$. Section 4 likewise uses the formula from Sect. 2 to compute the Chern-Simons-Witten invariants for torus bundles over $S^{1}$. Finally, Sect. 5 compares these values with the large $r$ asymptotic expansion from topological field theory (see [11]) and obtains explicit agreement.

\section{Formula for the Representation of $S L(2, \mathrm{Z})$}

\subsection{Preliminaries}

Any topological field theory associates to a surface $\Sigma_{g}$ of genus $g$ a representation of the mapping class group $\Gamma_{g}$ on a vector space $\mathscr{H}_{\Sigma}$, the "physical Hilbert space" of the theory. For the Chern-Simons theory, these representations may be obtained 
from conformal field theory, specifically from the Wess-Zumino-Witten model for the group $G$; there is a different representation of the mapping class group for each value of the level $k$, so we may denote the vector space by $\mathscr{H}_{\Sigma}^{(k)}$.

We shall pay particular attention to the case when $g=1$, so that one obtains representations of the torus mapping class group $S L(2, \mathbb{Z})$. These representations are known from the study of a space of theta functions arising in the theory of affine Lie algebras (see [21]): in fact (see [14], Appendix) they define representations of the modular group $P S L(2, \mathbb{Z})$. The representation is normally specified in terms of the generators $S, T$ of $P S L(2, \mathbb{Z})$ with

$$
S=\left[\begin{array}{cc}
0 & -1 \\
1 & 0
\end{array}\right], \quad T=\left[\begin{array}{ll}
1 & 1 \\
0 & 1
\end{array}\right]
$$

satisfying the relations

$$
S^{2}=(S T)^{3}=1
$$

In the present section we specialize still further to the case $G=S U(2)$. In this case, the torus vector space $\mathscr{H}_{T^{2}}^{(k)}$ has dimension $k+1$.

Notation. We adopt the notation of [23]:

$$
r=k+2
$$

and

$$
\begin{gathered}
e(\alpha) \stackrel{\text { def }}{=} \exp (2 \pi i \alpha) \\
e_{n}(\alpha) \stackrel{\text { def }}{=} \exp \left(2 \pi i \frac{\alpha}{n}\right)
\end{gathered}
$$

Also,

$$
\zeta \stackrel{\text { def }}{=} \exp \frac{i \pi}{4}
$$

Convention on square roots. Throughout the following, if $z \in \mathbb{C}, \sqrt{z}$ will denote the branch that is positive on positive real numbers, with a branch cut along the negative real axis.

Proposition 2.1 ([14], (A.44)). The representation of $P S L(2, \mathbb{Z})$ on $\mathscr{H}_{T^{2}}{ }^{(k)}$ is given by

$$
\begin{gathered}
S_{j l}=\sqrt{\frac{2}{r}} \sin \left(\frac{j l \pi}{r}\right)=\frac{1}{2 i} \sqrt{\frac{2}{r}}\left(e_{2 r}(j l)-e_{2 r}(-j l)\right), \\
T_{j l} \stackrel{\text { def }}{=} T_{j} \delta_{j l}, \quad T_{j}=\zeta^{-1} e_{4 r}\left(j^{2}\right) .
\end{gathered}
$$

Here, the indices $j, l$ have been chosen to run from 1 to $r-1$. For a general group $G$, the analogous representation is given in Proposition 4.2.

Lemma 2.2. These coefficients have the following symmetries:

$$
S_{j l}=S_{j(2 r+l)}=-S_{j(2 r-l)}, \quad T_{j l}=T_{j(2 r+l)}=T_{j(2 r-l)} .
$$

We wish first to derive an explicit formula for this representation of $S L(2, \mathbb{Z})$, which expresses the representation of an arbitrary element $U$ without expanding $U$ in terms of the generators $S$ and $T$. To derive the formula for the representation, we shall need the following classical number theoretic result: 
Proposition 2.3 (Gauss sum reciprocity formula in one dimension).

$$
\sum_{\lambda(\bmod n)} e_{2 n}\left(m \lambda^{2}\right) e(\psi \lambda)=\sqrt{\frac{i n}{m}} \sum_{\lambda(\bmod n)} e_{2 m}\left(-n(\lambda+\psi)^{2}\right),
$$

if $\lambda \in \mathbb{Z}, n, m \in \mathbb{Z}, n m$ is even and $n \psi \in \mathbb{Z}$.

This proposition is proved by a minor generalization of the argument given in [7] (Chap. IX, Theorem 1); the basic idea is to consider a limiting case of the Poisson summation formula applied to a Gaussian function. A more general version is given below (Proposition 4.3).

We now use the reciprocity formula for Gauss sums to derive an explicit formula for the representation $\mathscr{B}$ of $P S L(2, \mathbb{Z})$ which was specified in $(2.3)$. We are able to write

$$
\mathscr{R}(U), \quad U=\left[\begin{array}{ll}
a & b \\
c & d
\end{array}\right]
$$

in terms of $a, b, c, d$, starting with the expression for the element $U$ of $P S L(2, \mathbb{Z})$ in terms of the generators $S$ and $T$.

\subsection{Formula for the Representation}

Definition 2.4. Suppose $U \in S L(2, \mathbb{Z})$. A continued fraction expansion $\left[m_{1}, \ldots, m_{t}\right]$ for $U$ is a sequence of integers $m_{1}, \ldots, m_{t}$ such that

$$
U=T^{m_{t}} S \ldots T^{m_{1}} S \text {. }
$$

Such an expansion always exists, but is not unique because of the relations (2.2). The following proposition makes clear the reason for the name.

Proposition 2.5. Suppose

$$
U=\left[\begin{array}{ll}
a & b \\
c & d
\end{array}\right] \in S L(2, \mathbb{Z})
$$

and suppose $\left[m_{1}, \ldots, m_{t}\right]$ is a continued fraction expansion for $U$. Then

$$
a / c=m_{t}-\frac{1}{m_{t-1}-\frac{1}{\ldots-\frac{1}{m_{1}}}} .
$$

$$
b / a=-\left(\frac{1}{a_{1}}+\frac{1}{a_{2} a_{1}}+\ldots+\frac{1}{a_{t} a_{t-1}}\right) .
$$

Moreover, define $a_{i}, b_{i}, c_{i}, d_{i}$ by the partial evaluation of this product (for $i \geq 1$ ):

$$
\left[\begin{array}{cc}
a_{i} & b_{i} \\
c_{i} & d_{i}
\end{array}\right]=T^{m_{\imath}} S \ldots T^{m_{1}} S
$$

with the convention that

$$
a_{0}=d_{0}=1, \quad b_{0}=c_{0}=0 .
$$

Then these satisfy the recurrence relations (for $t \geq 2$ )

$$
\begin{array}{ll}
a_{t}=m_{t} a_{t-1}-c_{t-1}, & c_{t}=a_{t-1} ; \\
b_{t}=m_{t} a_{t-1}-d_{t-1}, & d_{t}=b_{t-1} .
\end{array}
$$


Proof. We first consider

$$
T^{m_{1}} S=\left[\begin{array}{ll}
a_{1} & b_{1} \\
c_{1} & d_{1}
\end{array}\right]=\left[\begin{array}{cc}
1 & m_{1} \\
0 & 1
\end{array}\right]\left[\begin{array}{cc}
0 & -1 \\
1 & 0
\end{array}\right]=\left[\begin{array}{cc}
m_{1} & -1 \\
1 & 0
\end{array}\right]
$$

so indeed the induction begins correctly. We then evaluate

$$
\left[\begin{array}{cc}
a_{t} & b_{t} \\
c_{t} & d_{t}
\end{array}\right]=\left[\begin{array}{cc}
m_{t} a_{t-1}-c_{t-1} & m_{t} b_{t-1}-d_{t-1} \\
a_{t-1} & b_{t-1}
\end{array}\right]
$$

which gives the inductive formulae (iii), (iv). Formula (iii) is clearly solved by the continued fraction expansion (i). As for (ii), observe that indeed $b_{1} / a_{1}=-1 / m_{1}$. By induction,

$$
\begin{aligned}
\left(\frac{1}{a_{1}}+\ldots+\frac{1}{a_{t} a_{t-1}}\right) & =-\frac{b_{t-1}}{a_{t-1}}+\frac{1}{a_{t} a_{t-1}} \\
& =\frac{-a_{t} b_{t-1}+1}{a_{t} a_{t-1}} \\
& =\frac{-\left(m_{t} a_{t-1}-c_{t-1}\right) b_{t-1}+1}{a_{t} a_{t-1}} \quad \text { (by (iii)) } \\
& \left.=\frac{-m_{t} a_{t-1} b_{t-1}+a_{t-1} d_{t-1}}{a_{t} a_{t-1}} \quad \text { (as } 1-c b=a d\right) \\
& =\frac{-m_{t} b_{t-1}+d_{t-1}}{a_{t}}=\frac{b_{t}}{a_{t}} \quad \text { (by (iv)) },
\end{aligned}
$$

completing the proof.

Lemma 2.6. Denote by $\mathscr{S}_{t}$ the sum

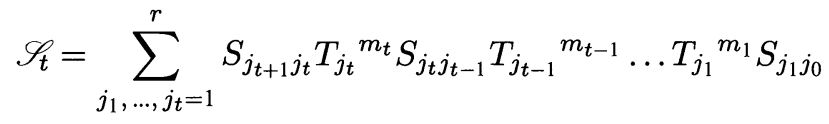

(in terms of the quantities $T_{j}, S_{j l}$ specified in (2.3)). Then in the notation of Proposition 2.5 , we have (provided that none of $a_{1}, \ldots, a_{t}$ vanish):

$$
\mathscr{I}_{t}=C_{t} \sum_{\substack{\gamma\left(\bmod 2 r a_{t}\right) \\ \gamma=j_{t+1}(\bmod 2 r)}}\left\{e_{4 r a_{t}}\left(-c_{t}\left(\gamma+\frac{j_{0}}{c_{t}}\right)^{2}\right)-e_{4 r a_{t}}\left(-c_{t}\left(\gamma-\frac{j_{0}}{c_{t}}\right)^{2}\right)\right\}
$$

where $C_{t}$ is given by

$$
C_{t}=i^{(t-1)} \zeta^{D_{t}}\left(-\frac{1}{\sqrt{2 r\left|a_{t}\right|}}\right) \zeta^{-\left(m_{1}+\ldots+m_{t}\right)} e_{4 r}\left\{-\left(\frac{1}{a_{1}}+\ldots+\frac{1}{a_{t-2} a_{t-1}}\right) j_{0}^{2}\right\}
$$

and $D_{t}=\operatorname{sgn}\left(a_{0} a_{1}\right)+\ldots+\operatorname{sgn}\left(a_{t-1} a_{t}\right)$.

Proof. First observe that each of the indices $j_{1}, \ldots, j_{t}$ appears in two of the $S$ in (2.5), so we may divide by appropriate factors of 2 and replace by a sum over 
$j_{1}, \ldots, j_{t}$ running from 1 to $2 r$ (using the symmetries in Lemma 2.2.) First we do not the calculation for $\mathscr{S}_{1}$ :

$$
\begin{aligned}
\mathscr{T}_{1}= & \frac{1}{2} \sum_{j_{1}=1}^{2 r} S_{j_{2} j_{1}} T_{j_{1}}{ }^{m_{1}} S_{j_{1} j_{0}} \\
= & \frac{1}{2} \cdot \frac{2}{r} \cdot \frac{1}{(2 i)^{2}} \cdot 2 \cdot \zeta^{-m_{1}} \cdot \sum_{j(\bmod 2 r)} e_{4 r}\left(m_{1} j^{2}\right) \\
& \times\left\{e_{2 r}\left\{\left(j_{2}+j_{0}\right) j\right\}-e_{2 r}\left\{\left(j_{2}-j_{0}\right) j\right\}\right\} .
\end{aligned}
$$

(In the large curly brackets, two terms corresponding to the complex conjugates of the terms shown have been removed, and the overall expression multiplied by 2 : this results from the substitution $j \rightarrow-j$.)

Now we apply the reciprocity formula (2.4): denoting the constant outside the second sum in (2.7) by $B_{1}$, we have

$$
\begin{aligned}
\mathscr{S}_{1} & =C_{1} \sum_{\beta\left(\bmod m_{1}\right)}\left\{e_{2 m_{1}}\left(-2 r\left(\beta+\frac{j_{2}+j_{0}}{2 r}\right)^{2}\right)-e_{2 m_{1}}\left(-2 r\left(\beta+\frac{j_{2}-j_{0}}{2 r}\right)^{2}\right)\right\} \\
& =C_{1} \sum_{\beta\left(\bmod m_{1}\right)}\left\{e_{4 r m_{1}}\left(-\left(2 r \beta+j_{2}+j_{0}\right)^{2}\right)-e_{4 r m_{1}}\left(-\left(2 r \beta+j_{2}-j_{0}\right)^{2}\right)\right\},
\end{aligned}
$$

where

$$
C_{1}=B_{1} \sqrt{\frac{2 r i}{m_{1}}}=-\frac{1}{\sqrt{2 r}} \sqrt{\frac{i}{a_{1}}} \zeta^{-m_{1}} .
$$

This confirms the first step in the induction.

Now we assume the result of the lemma inductively and write (again using the symmetries in Lemma 2.2 to expand the sum over $j_{t}$ to run from 1 to $2 r$ )

$$
\begin{aligned}
\mathscr{T}_{t}= & \frac{1}{2} \sum_{j_{t}=1}^{2 r} S_{j_{t+1} j_{t}} T_{j_{t}} m_{t} \cdot \mathscr{S}_{t-1} \\
= & \frac{1}{2} \frac{1}{2 i} \sqrt{\frac{2}{r}} C_{t-1} \sum_{j_{t}(\bmod 2 r)} \sum_{\substack{\gamma\left(\bmod 2 r a_{t-1)} \\
\gamma=j_{t}(\bmod 2 r)\right.}} \\
& \times\left\{e_{2 r}\left(j_{t+1} j_{t}\right)-e_{2 r}\left(-j_{t+1} j_{t}\right)\right\} \zeta^{-m_{t}} e_{4 r a_{t-1}}\left(m_{t} a_{t-1} j_{t}^{2}\right) \\
& \times e_{4 r a_{t-1}}\left(-c_{t-1} \gamma^{2}\right)\left\{e_{2 r a_{t-1}}\left(-\gamma j_{0}\right)-e_{2 r a_{t-1}}\left(\gamma j_{0}\right)\right\} e_{4 r a_{t-1} c_{t-1}}\left(-j_{0}^{2}\right) .
\end{aligned}
$$

We may now replace $j_{t}$ by $\gamma$ throughout the previous expression. Thus

$$
\begin{aligned}
\mathscr{S}_{t}= & \frac{1}{4 i} \sqrt{\frac{2}{r}} \zeta^{-m_{t}} e_{4 r a_{t-1} c_{t-1}}\left(-j_{0}^{2}\right) C_{t-1} \\
& \times \sum_{\gamma\left(\bmod 2 r c_{t}\right)}\left\{e_{2 r}\left(j_{t+1} \gamma\right)-e_{2 r}\left(-j_{t+1} \gamma\right)\right\} \\
& \times e_{4 r c_{t}}\left(m_{t} a_{t-1} \gamma^{2}\right) e_{4 r c_{t}}\left(-c_{t-1} \gamma^{2}\right)\left\{e_{2 r c_{t}}\left(-\gamma j_{0}\right)-e_{2 r c_{t}}\left(\gamma j_{0}\right)\right\}
\end{aligned}
$$


Now we combine the coefficients of the $\gamma^{2}$ factors using Proposition 2.5(iii), to obtain

$$
\begin{aligned}
\mathscr{S}_{t}= & \frac{1}{4 i} \sqrt{\frac{2}{r}} \zeta^{-m_{t}} e_{4 r a_{t-1} c_{t-1}}\left(-j_{0}^{2}\right) C_{t-1}(-2) \\
& \times \sum_{\gamma\left(\bmod 2 r c_{t}\right)} e_{4 r c_{t}}\left(a_{t} \gamma^{2}\right) \\
& \times\left\{e_{2 r c_{t}}\left(c_{t} j_{t+1}+j_{0}\right) \gamma-e_{2 r c_{t}}\left(c_{t} j_{t+1}-j_{0}\right) \gamma\right\} .
\end{aligned}
$$

(Here, the factor $(-2)$ immediately in front of the sum arises, as in the calculation of (2.7), from the substitution $\gamma \rightarrow-\gamma$ which allows us to condense four terms to two.)

Now another application of the reciprocity law (2.4) yields (denoting the constant in front of the sum in (2.9) by $B_{t}$ ),

$$
\begin{aligned}
\mathscr{S}_{t}= & \sqrt{\frac{2 r c_{t} i}{a_{t}}} B_{t} \sum_{\beta\left(\bmod a_{t}\right)}\left\{e_{2 a_{t}}\left(-2 r c_{t}\left(\beta+\frac{c_{t} j_{t+1}+j_{0}}{2 r c_{t}}\right)^{2}\right)+\right. \\
- & \left.e_{2 a_{t}}\left(-2 r c_{t}\left(\beta+\frac{c_{t} j_{t+1}-j_{0}}{2 r c_{t}}\right)^{2}\right)\right\} \\
= & \sqrt{\frac{2 r c_{t} i}{a_{t}}} B_{t} \sum_{\substack{\gamma\left(\bmod 2 r a_{t}\right) \\
\gamma=j_{t+1}(\bmod 2 r)}} \\
& \times\left\{e_{4 r a_{t}}\left(-c_{t}\left(\gamma+\frac{j_{0}}{c_{t}}\right)^{2}\right)-e_{4 r a_{t}}\left(-c_{t}\left(\gamma-\frac{j_{0}}{c_{t}}\right)^{2}\right)\right\},
\end{aligned}
$$

using $\gamma=2 r \beta+j_{t+1}$. This completes the induction.

By induction the formula for $C_{t}$ is

$$
C_{t}=i \sqrt{\frac{a_{t-1} i}{a_{t}}} \zeta^{-m_{t}} e_{4 r a_{t-1} c_{t-1}}\left(-j_{0}^{2}\right) C_{t-1}
$$

Notice that the phase of $\sqrt{a_{t-1} i / a_{t}}$ is $\zeta$ if $a_{t}, a_{t-1}$ have the same sign and $\zeta^{-1}$ if they have different signs. Thus these factors multiply to give a factor $\zeta^{D_{t}} /\left|a_{t}\right|^{1 / 2}$, where

$$
D_{t}=\operatorname{sgn}\left(a_{0} a_{1}\right)+\ldots+\operatorname{sgn}\left(a_{t-1} a_{t}\right) .
$$

So we have that

$$
\begin{aligned}
C_{t}= & i^{(t-1)} \zeta^{D_{t}}\left(-\frac{1}{\sqrt{2 r\left|a_{t}\right|}}\right) \zeta^{-\left(m_{1}+\ldots+m_{t}\right)} \\
& \times e_{4 r}\left\{-\left(\frac{1}{a_{0} a_{1}}+\ldots+\frac{1}{a_{t-2} a_{t-1}}\right) j_{0}^{2}\right\}
\end{aligned}
$$

as claimed. 
It is ndw easy to derive the formula for $\mathscr{B}(U)$. We have

$$
\begin{aligned}
& \mathscr{R}(U)_{j_{t} j_{0}}=T_{j_{t}}^{m_{t}} \mathscr{T}_{t-1} \\
& =\zeta^{-m_{t}} e_{4 r a_{t-1} c_{t-1}}\left(-j_{0}^{2}\right) C_{t-1} \sum_{\substack{\gamma\left(\bmod 2 r c_{t}\right) \\
\gamma=j_{t}(\bmod 2 r)}} e_{4 r a_{t-1}}\left(m_{t} a_{t-1} \gamma^{2}\right) \\
& \times e_{4 r a_{t-1}}\left(-c_{t-1} \gamma^{2}\right)\left\{e_{2 r a_{t-1}}\left(-\gamma j_{0}\right)-e_{2 r a_{t-1}}\left(\gamma j_{0}\right)\right\} \\
& =-\zeta^{-m_{t}} e_{4 r a_{t-1} a_{t-2}}\left(-j_{0}^{2}\right) C_{t-1} \sum_{\substack{\gamma\left(\bmod 2 r c_{t}\right) \\
\gamma=j_{t}(\bmod 2 r)}} e_{4 r a_{t-1}}\left(a_{t} \gamma^{2}\right) \\
& \times\left\{e_{2 r a_{t-1}}\left(\gamma j_{0}\right)-e_{2 r a_{t-1}}\left(-\gamma j_{0}\right)\right\} \\
& =-\zeta^{-m_{t}} e_{4 r a_{t-1} a_{t-2}}\left(-j_{0}^{2}\right) e_{4 r a_{t} a_{t-1}}\left(-j_{0}^{2}\right) C_{t-1} \\
& \times \sum_{\substack{\gamma\left(\bmod 2 r c_{t}\right) \\
\gamma=j_{t}(\bmod 2 r)}}\left\{e_{4 r a_{t-1}}\left(a_{t}\left(\gamma+\frac{j_{0}}{a_{t}}\right)^{2}\right)-e_{4 r a_{t-1}}\left(a_{t}\left(\gamma-\frac{j_{0}}{a_{t}}\right)^{2}\right)\right\} .
\end{aligned}
$$

Now using Proposition 2.5(ii) for the prefactor involving $j_{0}^{2}$, we have

$$
\begin{aligned}
& \mathscr{B}(U)_{j_{t} j_{0}} \\
& =\left(-i K_{t}\right) \frac{1}{\sqrt{2 r|c|}} e_{4 r a}\left(b j_{0}{ }^{2}\right) \\
& \quad \times \sum_{\substack{\gamma\left(\bmod 2 r c_{t}\right) \\
\gamma=j_{t}(\bmod 2 r)}}\left\{e_{4 r a_{t-1}}\left(a_{t}\left(\gamma+\frac{j_{0}}{a_{t}}\right)^{2}\right)-e_{4 r a_{t-1}}\left(a_{t}\left(\gamma-\frac{j_{0}}{a_{t}}\right)^{2}\right)\right\}
\end{aligned}
$$

where

$$
\begin{aligned}
& K_{t}=i^{t-1} \zeta^{D_{t-1}} \zeta^{\left(-m_{1}-\ldots-m_{t}\right)} \quad(t \geq 2), \\
& K_{1}=\zeta^{-m_{1}} .
\end{aligned}
$$

Combining all the terms quadratic in $j_{0}$, and replacing $j_{t}$ by $j$ and $j_{0}$ by $l$, we get finally

Proposition 2.7 (a). The representation of $S L(2, \mathbb{Z})$ on $\mathscr{H}_{\Sigma}^{(k)}$ is given by

$$
\begin{aligned}
\mathscr{R}(U)_{j l}= & (-i K(U)) \frac{1}{\sqrt{2 r|c|}} e_{4 r c}\left(d l^{2}\right) \\
& \times \sum_{\substack{\gamma(\bmod 2 r c) \\
\gamma=j(\bmod 2 r)}} e_{4 r c}\left(a \gamma^{2}\right)\left\{e_{2 r c}(\gamma l)-e_{2 r c}(-\gamma l)\right\} .
\end{aligned}
$$

Here, $K(U)=K_{t}$, where $K_{t}$ was given by (2.14); it will be shown in Proposition 2.8 that $K_{t}$ depends only on $U$ and not on the choice of a particular continued fraction expansion for $U$.

We know from Lemma 2.2 that $\mathscr{B}(U)_{j l}$ depends on $l$ only $\bmod 2 r$. To rewrite our formula so that this is manifest, we consider

$$
\mathscr{S} \stackrel{\text { def }}{=} \sum_{\substack{\gamma(\bmod 2 r c) \\ \gamma=j(\bmod 2 r)}} e_{4 r c}\left(a \gamma^{2}+2 \gamma l+d l^{2}\right) .
$$


Now completing the square by introducing $\lambda=\gamma+d l$, we have

$$
\begin{aligned}
\mathscr{S} & =\sum_{\substack{\lambda(\bmod 2 r c) \\
\lambda=j+d l(\bmod 2 r)}} e_{4 r c}\left(a(\gamma+d l)^{2}+(2-2 a d) \gamma l+\left(d-a d^{2}\right) l^{2}\right) \\
& =\sum_{\substack{\lambda(\bmod 2 r c) \\
\lambda=j+d l(\bmod 2 r)}} e_{4 r c}\left(a \lambda^{2}\right) e_{4 r}\left(-b d l^{2}-2 b l j\right) .
\end{aligned}
$$

Thus our sum is

Proposition 2.7 (b). The representation of $S L(2, \mathbb{Z})$ on $\mathscr{H}_{\Sigma}^{(k)}$ is given by

$$
\begin{aligned}
\mathscr{B}(U)_{j l}= & -i K(U) \frac{1}{\sqrt{2 r|c|}}\left\{\sum_{\substack{\lambda(\bmod 2 r c) \\
\lambda=j+d l(\bmod 2 r)}} e_{4 r c}\left(a \lambda^{2}\right) e_{4 r}\left(-b d l^{2}-2 b l j\right)\right. \\
& \left.-\sum_{\substack{\lambda(\bmod 2 r c) \\
\lambda=j-d l(\bmod 2 r)}} e_{4 r c}\left(a \lambda^{2}\right) e_{4 r}\left(-b d l^{2}+2 b l j\right)\right\} .
\end{aligned}
$$

Here, the value of $K(U)$ will be given in Proposition 2.8 .

This formula depends on $j$ and $l$ only $\bmod 2 r$, as we know it must. ${ }^{1}$

\subsection{The Rademacher phi Function}

Rademacher ([33], p. 150) defines an integer valued function on $\operatorname{PSL}(2, \mathbb{Z})$ by

$$
\Phi\left[\begin{array}{ll}
a & b \\
c & d
\end{array}\right]= \begin{cases}\frac{a+d}{c}-12(\operatorname{sign} c) s(d,|c|) & \text { if } c \neq 0 \\
\frac{b}{d} & \text { if } c=0 .\end{cases}
$$

Here, for $c>0$ the Dedekind sum $s(d, c)$ is defined by

$$
\begin{aligned}
& s(0,1)=0, \\
& s(d, c)=\sum_{k=1}^{c-1}\left(\left(\frac{k}{c}\right)\right)\left(\left(\frac{d k}{c}\right)\right),
\end{aligned}
$$

where for a real number $x$,

$$
((x))= \begin{cases}0, & x \in \mathbb{Z} ; \\ x-[x]-\frac{1}{2}, & \text { otherwise } .\end{cases}
$$

The Dedekind sum has the alternative definition (for $c>0$ )

$$
s(d, c)=\frac{1}{4 c} \sum_{k=1}^{c-1} \cot \frac{\pi k}{c} \cot \frac{\pi d k}{c} .
$$

\footnotetext{
1 After we had completed our work, we learned of a very similar formula for a representation of $S L(2, \mathbb{Z})$ (Proposition 4.1 of [38]) obtained in a different context and with different methods
} 
(For further properties of the Dedekind sum, see [15], especially p. 18.) The function $\Phi$ is "almost" a homomorphism from $S L(2, \mathbb{Z})$ to $\mathbb{Z}$; more precisely, Rademacher proves that if $A^{\prime \prime}=A^{\prime} A$, then

$$
\Phi\left(A^{\prime \prime}\right)=\Phi(A)+\Phi\left(A^{\prime}\right)-3 \operatorname{sign}\left(c c^{\prime} c^{\prime \prime}\right) .
$$

In particular, consider $A=T^{m} S$, so $\Phi(A)=m$; by induction, defining $\Phi_{t}$ by

$$
\Phi_{t} \stackrel{\text { def }}{=} \Phi\left(T^{m_{t}} S \ldots T^{m_{1}} S\right)
$$

we find from (2.18)

$$
\begin{aligned}
\Phi_{t}-\Phi_{t-1} & =m_{t}-3 \operatorname{sign}\left(a_{t-1} c_{t-1}\right), \quad(t \geq 2) \\
\Phi_{1} & =m_{1} .
\end{aligned}
$$

Proposition 2.8. The quantity $K_{t}$ from (2.14) is given by

$$
K_{t}=\zeta^{-\Phi_{t}} \operatorname{sign}\left(c_{t}\right) \text {; }
$$

in other words we may define

$$
K(U)=K_{t}=\zeta^{-\Phi(U)} \operatorname{sign}(c) .
$$

Proof. The recurrence relation satisfied by $K_{t}$ is (from (2.14))

$$
K_{t}=\zeta^{-\Delta_{t}}
$$

where

$$
\begin{aligned}
\Delta_{t}-\Delta_{t-1} & =-2-\operatorname{sign}\left(c_{t-1} a_{t-1}\right)+m_{t} \quad(t \geq 2) \\
\Delta_{1} & =m_{1} .
\end{aligned}
$$

The discrepancy between this and (2.20) is $\Phi_{t}=\Delta_{t}+\sum_{i=2}^{t} n_{i}$, where

$$
n_{l}=2-2 \operatorname{sign}\left(c_{l-1} c_{l}\right)
$$

so

$$
\zeta^{n_{t}}=\operatorname{sign}\left(c_{t-1}\right) \operatorname{sign}\left(c_{t}\right) \quad(t \geq 2)
$$

and (since $\left.c_{1}=1\right)$

$$
\operatorname{sign} c_{t}=\zeta^{n_{2}+\ldots+n_{t}}
$$

\subsection{Framing Dependence}

The invariants $Z(M, r)$ depend on a choice of 2-framing $\pi$ for $M$ [2], i.e., on the choice of a homotopy equivalence class $\pi$ of trivializations of $T M \oplus T M$, twice the tangent bundle of $M$ viewed as a Spin(6) bundle. The possible 2-framings correspond to $\mathbb{Z}$. The identification with $\mathbb{Z}$ is through the signature defect $\delta(M, \pi)$ defined by ${ }^{2}$

$$
\delta(M, \pi)=\operatorname{sign}(X)-\frac{1}{6} p_{1}(2 T X, \pi),
$$

where $X$ is a 4-manifold with boundary $M$ and $p_{1}(2 T X, \pi)$ is the relative Pontrjagin number associated to the framing $\pi$ of the bundle $T X \oplus T X$. The canonical 2framing $\pi^{c}$ [2] corresponds to $\delta\left(M, \pi^{c}\right)=0$. When we wish to specify explicitly the

${ }^{2}$ Note that our identification (2.22) of 2-framings with $\mathbb{Z}$ differs from that in [11] by a minus sign 
dependence of $Z(M, r)$ on the framing $\pi$, we shall use the notation $Z(M, r ; \delta(M, \pi))$. We have the following

Lemma 2.9. If the two-framings $\pi, \pi^{\prime}$ satisfy $\delta\left(M, \pi^{\prime}\right)=\delta(M, \pi)+1$, then

$$
Z\left(M, r ; \delta\left(M, \pi^{\prime}\right)\right)=Z(M, r ; \delta(M, \pi)) e^{2 \pi i c / 24} .
$$

Here, $c$ is the central charge, which is defined by (A.4).

Remark. For $G=S U(2)$, we have exp $\frac{2 \pi i c}{24}=\exp \frac{i \pi}{4} \exp \left\{-\frac{i \pi}{2(k+2)}\right\}=\zeta e_{4 r}(-1)$.

If a three-manifold $M$ is specified by a framed link $L$ for surgery, there is a standard 2-framing $\pi_{L}$ associated to the surgery link [11]. This 2-framing differs from the canonical one by the following factor:

Proposition 2.10 ([11], Theorem 2.3). The signature defect of the 2-framing associated to a framed link $L$ with the framings of components specified by integers $m_{1}, \ldots, m_{t}$ is

for

$$
\delta\left(M, \pi_{L}\right)=\varphi_{L}
$$

$$
\varphi_{L}=3 \sigma\left(W_{L}\right)-\sum_{i=1}^{t} m_{i}
$$

where $\sigma\left(W_{L}\right)$ is the signature of the linking matrix $W_{L}$ of $L$.

\section{Lens Space Invariants}

In this section we apply our formula for the representation $\mathscr{B}$ of $S L(2, \mathbb{Z})$ to obtain a formula for Witten invariants of lens spaces $L(p, q)$. Our result for general $r$ is Theorem 3.4. In Sect. 3.2 we also provide formulas for the case $(r, p)=1$, where further simplifications are possible.

Our first formula (3.8) for lens space invariants is for a 2 -framing that arises naturally when one glues together solid tori using elements of $S L(2, \mathbb{Z})$. Theorem 3.4 is the result of correcting this formula to the canonical 2-framing of Atiyah [2]. Theorem 3.7 gives the specialization of the formula in Theorem 3.4 to the case when the integer parameter $r$ is prime to $p$.

\subsection{General Formula}

Let us now apply our formula for the representation $\mathscr{R}$ to obtain a formula for Witten invariants of lens spaces. The lens space $L(p, q)$ is specified by a pair $p, q$ of coprime integers with $0<|q|<p{ }^{3}$ in fact $l(p,-q)$ is diffeomorphic to $L(p, q)$ via an orientation reversing diffeomorphism. The space $L(p, q)$ is formed by surgery on $S^{3}$ along a framed link $L$ which is a chain with the framings of successive components specified by integers $m_{1}, \ldots, m_{t-1}$. Such a surgery is a $-p / q$ surgery on the unknot

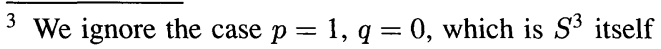


in $S^{3}$ (see' e.g. [11]): this means that a solid torus around the unknot is removed and glued back using a matrix

$$
A=\left[\begin{array}{cc}
p & d \\
-q & -b
\end{array}\right] \in S L(2, \mathbb{Z})
$$

Here, the matrix $A$ is given by a continued fraction expansion $\left[m_{1}, \ldots, m_{t-1}\right]$, i.e.,

$$
A=T^{m_{t-1}} S \ldots T^{m_{1}} S \quad \text { (recall Proposition 2.5). }
$$

In other words, the $m_{i}$ satisfy

$$
-p / q=m_{t-1}-\frac{1}{m_{t-2}-\frac{1}{\ldots-\frac{1}{m_{1}}}} .
$$

This surgery is obviously equivalent to gluing two standard solid tori using

$$
U=S A=\left[\begin{array}{ll}
q & b \\
p & d
\end{array}\right]
$$

thus $U$ has a continued fraction expansion $\left[m_{1}, \ldots, m_{t-1}, m_{t}=0\right]$.

Via Proposition 2.10 , there is a 2 -framing $\pi_{L}$ associated to this surgery description, with signature defect $\delta\left(M, \pi_{L}\right)=\varphi_{L}$. It is shown in [11] that $\varphi_{L}$ depends only on $A$ and not on the particular choice of integers $\left(m_{1}, \ldots, m_{t-1}\right){ }^{4}$ So we may introduce the notation

$$
\varphi_{L}=\tilde{\varphi}(A)
$$

Thus we have

$$
\tilde{\varphi}(A)=3 \operatorname{sign} W_{L}-\sum_{i=1}^{t-1} m_{\imath},
$$

where the linking matrix $W_{L}$ is given by

$$
W_{L}=\left(\begin{array}{ccccc}
m_{1} & 1 & 0 & \ldots & 0 \\
1 & m_{2} & 1 & \ldots & 0 \\
0 & 1 & \ldots & & \\
\vdots & 0 & 1 & \ldots & \\
& & & 1 & m_{t-1}
\end{array}\right)
$$

The lens space $L(p, q)$ is obtained by gluing two standard solid tori using $U$; thus, according to the TQFT axioms, the Witten invariant of $L(p, q)$ in the 2-framing $\tilde{\varphi}(-S U)$ is given by

$$
Z(L(p, q), r ; \tilde{\varphi}(-S U))=\mathscr{R}(U)_{11}
$$

\footnotetext{
4 This will also follow from Proposition 3.5, whose proof does not depend on it
} 
Using the expression (2.13), this is

$$
\begin{aligned}
Z(L(p, q), r ; \tilde{\varphi}(-S U))= & -i \frac{\zeta^{-\Phi(U)}}{\sqrt{2 r p}} e_{4 r q}(b) \sum_{\substack{\gamma(\bmod 2 r p) \\
\gamma=1(\bmod 2 r)}} \\
& \times\left\{e_{4 r p}\left(q\left(\gamma+\frac{1}{q}\right)^{2}\right)-e_{4 r p}\left(q\left(\gamma-\frac{1}{q}\right)^{2}\right)\right\} \\
= & -i \frac{\zeta^{-\Phi(U)}}{\sqrt{2 r p}} e_{4 r q}(b) \\
& \times \sum_{n=1}^{p}\left\{e_{4 r p q}(2 r n q+q+1)^{2}-e_{4 r p q}(2 r n q+q-1)^{2}\right\} \\
= & -i \frac{\zeta^{-\Phi(U)}}{\sqrt{2 r p}} e_{4 r q}(b) \sum_{n=1}^{p} e_{p}\left(q r n^{2}\right) \\
& \times\left\{e_{p}(n(q+1)) e_{4 r p q}(q+1)^{2}-e_{p}(n(q-1)) e_{4 r p q}(q-1)^{2}\right\} .
\end{aligned}
$$

We now wish to obtain a formula for the lens space Witten invariant in the canonical framing. We need the following preliminary lemma:

Lemma 3.1. Assume $|p / q|>1$. Then $-p / q$ has a continued fraction expansion given by

$$
-p / q=m_{t-1}-\frac{1}{m_{t-2}-\frac{1}{\ldots-\frac{1}{m_{1}}}},
$$

with all $m_{i} \geq 2$ or with all $m_{i} \leq-2$.

Proof. Assume first that $-p / q>0$. By induction, any rational number $>1$ has such a continued fraction expansion. Indeed, set $m_{t-1}$ to be the least integer $>m / n$, so $m / n=m_{t-1}-\left(m^{\prime} / n^{\prime}\right)^{-1}$, where $m^{\prime}=n<m$ and $1 \leq n^{\prime}<m^{\prime}$. Since the denominators continue to decrease, this process must terminate. It follows likewise that a rational number $<-1$ has a continued fraction expansion with all $m_{i} \leq-2$.

In this situation, the signature defect is related to the Rademacher phi function by the following lemma.

Lemma 3.2. If $A$ is given by a continued fraction expansion (3.2) for $-p / q$ with all $m_{i} \geq 2$ or all $m_{i} \leq-2$, then

$$
\Phi(S A)=-\tilde{\varphi}(A) .
$$

Proof. The linking matrix $W_{L}$ has signature $t-1$ if all $m_{i} \geq 2$ ([16], Lemma 8.12). Thus $\tilde{\varphi}(A)=3 t-3-\left(m_{1}+\ldots+m_{t-1}\right)$ (from Proposition 2.10). In this case, all the $a_{i}$ are also $\geq 1$ (see the proof of Lemma 3.1, and (2.5) for the definition of the $a_{i}$.) Thus (2.20) shows $\Phi(S A)$ is equal to $-\tilde{\varphi}(A)$. A minor alteration of this argument makes it work also for the case $-p / q<0$.

We may now reduce to the Witten invariant of $L(p, q)$ in the canonical 2-framing: 
Lemma 3.3. The quantity

$$
\begin{aligned}
& \left(e_{4 r}(-1) \zeta\right)^{\Phi(U)} Z(L(p, q), r ; \tilde{\varphi}(-S U)) \\
& =-i e_{4 r}(-\Phi(U)) \frac{1}{\sqrt{2 r p}} e_{4 r q}(b) \sum_{ \pm} \sum_{n=1}^{p} \pm e_{p}\left(q r n^{2}\right) \\
& \quad \times e_{p}(n(q \pm 1)) e_{4 r p q}(q \pm 1)^{2}
\end{aligned}
$$

depends only on $p$ and on $q(\bmod p)$ : it is independent of the choice of $U$.

Proof. The factors in (3.11) involving roots of unity of order some multiple of $r$ give (recalling that $a=q, c=p$ )

$$
e_{4 r p q}\left(p b+q^{2} \pm 2 q+1\right)=e_{4 r p}(d+q \pm 2) .
$$

Introduce the integer $q^{*}(\bmod p)$ solving $q^{*} q=1(\bmod p)$. Now notice using the Definition (2.15) of $\Phi(U)$ that

$$
e_{4 r p}(d+q \pm 2) e_{4 r}(-\Phi(U))=e_{2 r p}( \pm 1) e_{4 r}\left(12 s\left(q^{*}, p\right)\right)=e_{2 r p}( \pm 1) e_{4 r}(12 s(q, p))
$$

since $d=q^{*}(\bmod p)$ and $s\left(q^{*}, p\right)=s(q, p)$. This gives

$$
\begin{aligned}
& \left(e_{4 r}(-1) \zeta\right)^{\Phi(U)} Z(L(p, q), r ; \tilde{\varphi}(-S U)) \\
& \quad=-\frac{i}{\sqrt{2 r p}} e_{4 r}(12 s(q, p)) \sum_{ \pm} \sum_{n=1}^{p} \pm e_{2 r p}( \pm 1) e_{p}\left(q r n^{2}\right) e_{p}(n(q \pm 1)) .
\end{aligned}
$$

The right-hand side depends only on $p$ and on $q(\bmod p)$.

Hence, finally, we have

Theorem 3.4. The Witten invariant of the lens space $L(p, q)$ in the canonical 2-framing is given by

$$
Z(L(p, q), r ; 0)=-\frac{i}{\sqrt{2 r p}} e_{4 r}(12 s(q, p)) \sum_{ \pm} \sum_{n=1}^{p} \pm e_{2 r p}( \pm 1) e_{p}\left(q r n^{2}\right) e_{p}(n(q \pm 1)) .
$$

Proof. If we choose $U$ so that $A=-S U$ satisfies the hypothesis of Lemma 3.2, then that lemma says the factor $\left(e_{4 r}(-1) \zeta\right)^{\Phi(U)}$ in $(3.13)$ is just the factor $\left(e_{4 r}(-1) \zeta\right)^{-\tilde{\varphi}(-S U)}$ that is picked up when one changes from the framing $\tilde{\varphi}(-S U)$ to the canonical framing (see Lemma 2.9), so (3.13) represents the value $Z(L(p, q), r ; 0)$ in the canonical framing.

We may now use Lemma 3.3 to generalize the result of Lemma 3.2 to arbitrary $U \in S L(2, \mathbb{Z})$. In other words, we obtain a formula for the Rademacher phi function $\Phi(U)$ in terms of the signature of a matrix associated to a continued fraction expansion of $U$; this formula was obtained in [24]. We show that the formula follows immediately from our results, using the transformation properties (Lemma 2.9) of the Witten invariant under change of framing, and the identification (Proposition 2.10) of the signature defect of the framing associated to a framed link. We have

Proposition 3.5. For arbitrary $A \in S L(2, \mathbb{Z})$,

$$
\Phi(S A)=-\tilde{\varphi}(A)
$$

where $\tilde{\varphi}(A)$ is given by (3.5). 
Proof. The formula (3.13) expresses

$$
Z(L(p, q), r ; \tilde{\varphi}(-S U)) \quad \text { as } Z(L(p, q), r ; 0) \times\left(\zeta e_{4 r}(-1)\right)^{-\Phi(U)} .
$$

But by Lemma 2.9 we also have

$$
Z(L(p, q), r ; \tilde{\varphi}(-S U))=Z(L(p, q), r ; 0) \times\left(\zeta e_{4 r}(-1)\right)^{\tilde{\varphi}(-S U)},
$$

where $\tilde{\varphi}(A)$ was specified by (3.5).

\subsection{The Case $(r, p)=1$}

We now rewrite our formula (Theorem 3.4) for the lens space invariants in a more transparent form when $(p, r)=1$. Formulas of the type obtained in this section for the Witten invariant of $L(p, q)$ have been obtained independently by Garoufalidis [13] and Kirby and Melvin [25], who work under the assumption that $r$ is prime to $p$. In this section we assume $q, r>0$. Consider first the factor

$$
\begin{aligned}
\mathscr{S} & =\sum_{ \pm} \pm \sum_{n=1}^{p} e_{2 r p}( \pm 1) e_{p}\left(q r n^{2}\right) e_{p}(n(q \pm 1)) \\
& =\sum_{ \pm} \pm e_{2 r p}( \pm 1) \sum_{n=1}^{p} e_{p}\left(q r\left(n+\frac{q \pm 1}{2 q r}\right)^{2}\right) e_{p}\left(-q r\left(\frac{q \pm 1}{2 q r}\right)^{2}\right) .
\end{aligned}
$$

Case 1: $p$ odd. We can then find integers $l$ and $h$ such that

$$
p l+4 q r h=1 .
$$

Defining $\nu_{ \pm}=q \pm 1$, and completing the square, we than get

$$
\begin{aligned}
\mathscr{S} & =\sum_{ \pm} \pm e_{2 r p}( \pm 1) \sum_{n=1}^{p} e_{p}\left\{q r\left(n+2 \nu_{ \pm}\left(h-\frac{p l}{4 q r}\right)\right)\right\}^{2} e_{p}\left(-\frac{\nu_{ \pm}^{2}}{4 q r}\right) \\
& =\sum_{ \pm} \pm e_{2 r p}( \pm 1) \sum_{n=1}^{p} e_{p}\left(q r n^{2}\right) e_{p}\left(\frac{\nu_{ \pm}^{2}\left(p^{2} l^{2}-1\right)}{4 q r}\right) \\
& =\left(\frac{q r}{p}\right) G(1, p) \mathscr{S}^{\prime} .
\end{aligned}
$$

for

$$
\mathscr{S}^{\prime}=\sum_{ \pm} \pm e_{2 r p}( \pm 1) e_{p}\left\{\left(q^{2} \pm 2 q+1\right)(-2+4 q r h) h\right\} .
$$

Here, we have introduced the notation

$$
G(h, p)=\sum_{n=1}^{p} e_{p}\left(h n^{2}\right),
$$

and used the properties ([28], Chap. IV, Sect.3) that

Proposition 3.6. If $p$ is an odd integer $\geq 1$, than

$$
G(h, p)=\left(\frac{h}{p}\right) G(1, p),
$$

where $\left(\frac{h}{p}\right)$ is the Legendre symbol. 
The explicit values for $G(1, p)$ for arbitrary $p>0$ are given by

$$
G(1, p)=\sqrt{p} \times \begin{cases}1, & p=1(\bmod 4) \\ 0, & p=2(\bmod 4) \\ i, & p=3(\bmod 4) \\ (1+i) \sqrt{2}, & p=0(\bmod 4)\end{cases}
$$

We first decompose $\mathscr{S}^{\prime}$ as $\mathscr{S}^{\prime}=e^{\beta}\left(e^{\alpha}-e^{-\alpha}\right)$. Thus we have

$$
e^{\alpha}=e_{2 r p}(1+4 q r h(-2+4 q r h))=e_{2 r p}\left(p^{2} l^{2}\right)=e_{2 r}(l) .
$$

Also

$$
e^{\beta}=e_{p}\left(\left(q^{2}+1\right) h(-1-p l)\right)=e_{p}\left(-h\left(q^{2}+1\right)\right) .
$$

Now because of $(3.15), h$ is $4^{*} q^{*} r^{*}(\bmod p)$, so we have

$$
e^{\beta}=e_{p}\left(-4^{*}\left(q+q^{*}\right) r^{*}\right) \text {. }
$$

(We have introduced the notation $q^{*}$ for $q^{-1}(\bmod p)$ )

Case 2: $p$ even. In this case, $q$ is odd and we define the integers $\nu_{ \pm}=(q \pm 1) / 2$. We choose integers $l$ and $h$ satisfying

$$
4 p l+q r h=1 .
$$

Then, as before, we have

$$
\begin{aligned}
\mathscr{S} & =\sum_{ \pm} \pm e_{2 r p}( \pm 1) \sum_{n=1}^{p} e_{p}\left(q r\left\{n+\nu_{ \pm}\left(h-\frac{4 p l}{q r}\right)\right\}^{2}\right) e_{p}\left(-\frac{\nu_{ \pm}^{2}}{q r}\right) \\
& =\sum_{n=1}^{p} e_{p}\left(q r n^{2}\right) \sum_{ \pm} \pm e_{2 r p}( \pm 1) e_{p q r}\left\{\nu_{ \pm}^{2}\left(16 p^{2} l^{2}-1\right)\right\} .
\end{aligned}
$$

We manipulate the Gauss sum using the reciprocity formula (2.4); we take $p=2^{\alpha} p^{\prime}$ where $p^{\prime}$ is odd, and consider

$$
\sum_{n=1}^{p} e_{p}\left(q r n^{2}\right)=2 \sum_{n=1}^{2^{\alpha-1} p^{\prime}} \exp \frac{i \pi q r n^{2}}{2^{\alpha-1} p^{\prime}}
$$

This sum is zero if $\alpha=1$ (i.e., if $p=2(\bmod 4))$. Otherwise, if $p=0(\bmod 4)$, reciprocity (2.4) shows

$$
\begin{aligned}
\sum_{n=1}^{p} e_{p}\left(q r n^{2}\right) & =e_{8}(1) \sqrt{\frac{2 p}{q r}} \sum_{n=1}^{q r} \exp -\frac{i \pi 2^{\alpha-1} p^{\prime} n^{2}}{q r} \\
& =\sqrt{2 p} e_{8}(1)\left(\frac{2^{\alpha-2} p^{\prime}}{q r}\right) \times \begin{cases}1, & q r=1(\bmod 4) \\
-i, & q r=3(\bmod 4) .\end{cases}
\end{aligned}
$$

We also have to treat the factor

$$
\mathscr{S}^{\prime}=\sum_{ \pm} \pm e_{2 r p}( \pm 1) e_{p q r}\left\{\nu_{ \pm}^{2}\left(16 p^{2} l^{2}-1\right)\right\}
$$

This is

$$
\mathscr{S}^{\prime}=\sum_{ \pm} \pm e_{2 r p}( \pm 1) e_{4 p}\left\{\left(q^{2} \pm 2 q+1\right)(-2+q r h) h\right\}
$$


We get $\mathscr{S}^{\prime}=e^{\beta}\left(e^{\alpha}-e^{-\alpha}\right)$, where

$$
e^{\alpha}=e_{2 r p}\{(1-4 p l)(-1-4 p l)+1\}=e_{r}(2 l(1-q r h))=e_{r}(2 l) .
$$

Now

$$
e^{\beta}=e_{4 p}\left\{\left(q^{2}+1\right)(-1-4 p l) h\right\}=e_{4 p}\left(-\left(q^{2}+1\right) h\right) .
$$

Thus we get simply

$$
e^{\beta}=e_{4 p}\left(-\left(q^{2}+1\right) q^{*} r^{*}\right)=e_{4 p}\left(-\left(q+q^{*}\right) r^{*}\right) .
$$

These results are summarized in the following theorem:

Theorem 3.7. When $r$ is prime to $p$, we have the following values for $Z(L(p, q), r)$ in the canonical framing:

p odd:

$$
Z=\sqrt{\frac{2}{r}}\left(\frac{q r}{p}\right) \sin \frac{\pi l}{r} e_{4 r}(12 s(q, p)) e_{p}\left(-\left(q+q^{*}\right) 4^{*} r^{*}\right) \varepsilon
$$

where

$$
\varepsilon=\left\{\begin{array}{ll}
1, & p=1(\bmod 4) \\
i, & p=3(\bmod 4)
\end{array} .\right.
$$

Here, $q^{*}, r^{*}$ are inverses of $q$ and $r$ with respect to $p$, and $l$ is the inverse of $p(\bmod 4 r)$.

$p=2 \bmod 4: Z=0$.

$p=0 \bmod 4$ :

$$
Z=\sqrt{2} \sqrt{\frac{2}{r}} \sin \frac{4 l \pi}{r}\left(\frac{p / 4}{q r}\right) e_{s}(1) e_{4 r}(12 s(q, p)) e_{4 p}\left(-\left(q+q^{*}\right) r^{*}\right) \delta
$$

where

$$
\delta=\left\{\begin{array}{ll}
1, & q r=1(\bmod 4) \\
-i, & q r=3(\bmod 4)
\end{array} .\right.
$$

Here, $4 l$ is the inverse of $p(\bmod r)$, and $q^{*}, r^{*}$ are inverses of $q, r(\bmod 4 p)$.

Remark 3.8. The identity $Z(L(p, q) ; r)=0$ when $p=2(\bmod 4)$ is not necessarily satisfied when $r$ is not coprime to $p$ (consider for example $L(6,1)$ ). Thus the case $(r, p)=1$ is not entirely typical of the behaviour of the invariants.

Remark 3.9. Distinguishing Lens Spaces. Notice that if $p$ is known, then by taking the $2 p^{\text {th }}$ or $4 p^{\text {th }}$ powers of the expressions given in Proposition 3.7 and varying over all $r$, one may use the values of $Z$ to extract the Dedekind sum $s(q, p)$. Since $s(q, p)=\left(q+q^{*}\right) / p(\bmod \mathbb{Z})($ see $(2.15))$, the value of $s(q, p)$ determines the value of $q+q^{*}(\bmod p)$. If $p$ is prime, this yields a quadratic equation over the field $\mathbb{Z}_{p}$, which one may solve for the pair $\left\{q, q^{*}\right\}$ : recall that the pair $\left\{q, q^{*}\right\}$ classifies the lens spaces $L(p, q)$ up to orientation preserving diffeomorphism. If $p$ is not prime, however, $\mathbb{Z}_{p}$ is not a field and so the values of $q+q^{*}(\bmod p)$ will not always uniquely determine $\left\{q, q^{*}\right\}$. (Kirby has pointed out that $L(65,8)$ and $L(65,18)$ are not distinguished by $Z(L(p, q), r ; 0)$, for $r$ prime to $p=65$ : this is because for $p=65$, the values $q=8$ and $q=18$ both give $q+q^{*}=0$.)

Remark 3.10. We should clarify one point regarding the Dedekind sum factors. According to [23] Sect. 1, the invariant

$$
\tau_{r}(M)=(\sqrt{2 / r} \sin (\pi / r))^{-1} Z(M, r ; 0)
$$


takes values in the ring $\mathbb{Z}\left[e_{8 r}( \pm 1), r^{-1}\right]$. This is not superficially obvious from Theorem 3.7: however, when $p$ is odd, a short calculation using (3.15) and the definition (2.15) of the Rademacher phi function establishes that

$$
e_{4 r}(12 s(q, p)) e_{p}\left(-\left(q+q^{*}\right) 4^{*} r^{*}\right)=e_{4 r}\left(q l-\Phi\left[\begin{array}{cc}
q & -l \\
p & 4 r h
\end{array}\right]\right) \text {. }
$$

Similarly if $p=0(\bmod 4)$ we have using (3.19)

$$
e_{4 r}(12 s(q, p)) e_{4 p}\left(-\left(q+q^{*}\right) r^{*}\right)=e_{4 r}\left(4 q l-\Phi\left[\begin{array}{cc}
q & -4 l \\
p & r h
\end{array}\right]\right) \text {. }
$$

Thus the invariants specified by Theorem 3.7 do indeed belong to the ring specified in [23].

\section{Torus Bundles over the Circle}

Recall that for a surface $\Sigma$ and a diffeomorphism $\beta: \Sigma \rightarrow \Sigma$, the mapping torus $\Sigma_{\beta}$ is defined as

$$
\Sigma_{\beta}=\Sigma \times[0,1] / \sim
$$

where

$$
(x, 0) \sim(\beta(x), 1) .
$$

The axioms of topological field theory say that in an appropriate 2-framing (see (4.4)),

$$
Z\left(\Sigma_{\beta}, r\right)=\operatorname{Tr} \mathscr{B}(\beta) .
$$

We shall particularly consider the case when $\Sigma$ is a 2-torus and $\beta$ is an element $U$ of $S L(2, \mathbb{Z})$.

\subsection{Torus Bundle Invariants for $G=S U(2)$}

In this section, we consider

$$
U=\left[\begin{array}{ll}
a & b \\
c & d
\end{array}\right] \in S L(2, \mathbb{Z}), \quad a+d \neq \pm 2 .
$$

We derive the $S U(2)$ Chern-Simons partition function of $\Sigma_{U}$, where $\Sigma$ is a 2-torus. There is a canonical 2-framing $\xi_{U}[1]$ associated to the turus bundle over $S^{1}$ formed using $U \in S L(2, \mathbb{Z})$. This framing has signature defect

$$
\psi(U)=-\Phi(U)+3 \operatorname{sign}(c(a+d)) .
$$

According to the axioms of topological field theory, we have

$$
Z\left(\Sigma_{U}, r ; \psi(U)\right)=\operatorname{Tr} \mathscr{R}(U) .
$$

Thus, using Proposition 2.7(a), the Witten invariant is

$$
\begin{aligned}
Z\left(\Sigma_{U}, r ; \psi(U)\right)= & \frac{1}{2|c|} \sum_{j=1}^{2 r|c|} \mathscr{R}(U)_{j j} \\
= & \operatorname{sign}(c) \zeta^{-\Phi(U)} \sqrt{\frac{2}{r|c|}} \cdot \frac{1}{4 i|c|} \cdot \sum_{ \pm} \pm \sum_{\beta \bmod c} \sum_{j \bmod 2 r|c|} e_{4 r c}\left(d j^{2}\right) \\
& \times e_{4 r c}\left(a(j+2 r \beta)^{2}\right) e_{2 r c}( \pm(j+2 r \beta) j) .
\end{aligned}
$$


We may now replace the summation variable $j$ by $j-r \beta$, yielding

$$
\begin{aligned}
Z\left(\Sigma_{U}, r ; \psi(U)\right)= & \frac{1}{4 i|c|} \operatorname{sign}(c) \zeta^{-\Phi(U)} \sqrt{\frac{2}{r|c|}} \sum_{j(\bmod 2 r|c|)} \sum_{ \pm} \pm \sum_{\beta(\bmod c)} \\
& \times e_{4 r c}\left(d(j-r \beta)^{2}\right) e_{4 r c}\left(a(j+r \beta)^{2}\right) e_{2 r c}( \pm(j+r \beta)(j-r \beta)) \\
= & \frac{1}{4 i|c|} \operatorname{sign}(c) \zeta^{-\Phi(U)} \sqrt{\frac{2}{r|c|}} \sum_{j, \beta, \pm} \pm e_{4 r c}\left((d+a \pm 2) j^{2}\right) \\
& \times e_{2 c}((a-d) j \beta) e_{4 c}\left(r(d+a \mp 2) \beta^{2}\right) .
\end{aligned}
$$

Applying the reciprocity formula (2.4) to the sum over $j$, we get

$$
\begin{aligned}
Z\left(\Sigma_{U}, r ; \psi(U)\right)= & \sum_{ \pm} \pm \frac{1}{4 i|c|} \operatorname{sign}(c) \zeta^{-\Phi(U)} \sqrt{\frac{2}{r|c|} \sqrt{\frac{i(2 r c)}{d+a \pm 2}}} \\
& \times \sum_{\gamma=1}^{|d+a \pm 2|} \sum_{\beta(\bmod c)} e_{d+a \pm 2}\left(-r c\left(\gamma+\frac{(a-d) \beta}{2 c}\right)^{2}\right) \\
& \times e_{4 c}\left(r(d+a \mp 2) \beta^{2}\right) .
\end{aligned}
$$

The coefficient of $\beta^{2}$ in the exponential is

$$
2 \pi i \cdot \frac{r}{4 c(d+a \pm 2)}\left\{(d+a)^{2}-4-(d-a)^{2}\right\}=2 \pi i \cdot \frac{r}{4 c(d+a \pm 2)}(4 b c),
$$

so we obtain finally

$$
\begin{aligned}
& Z\left(\Sigma_{U}, r ; \psi(U)\right) \\
& =\sum_{ \pm} \pm \frac{1}{2 i|c| \sqrt{|d+a \pm 2|}} \operatorname{sign}(c) \zeta^{-\Phi(U)+3 \operatorname{sign}(c(d+a \pm 2))} i^{-\operatorname{sign}(c(d+a \pm 2))} \\
& \quad \times \sum_{\beta(\bmod c)} \sum_{\gamma=1}^{|d+a \pm 2|} \exp 2 \pi i r \frac{-c \gamma^{2}+(a-d) \gamma \beta+b \beta^{2}}{d+a \pm 2}
\end{aligned}
$$

(In the last expression, the sign of the $\gamma \beta$ term was changed by substituting $-\beta$ for $\beta$.) For simplicity, we restrict to the case when $U$ is hyperbolic, i.e., $|a+d|>2$. Then, correcting to the canonical framing, we have

$$
\begin{aligned}
Z\left(\Sigma_{U}, r ; 0\right)= & e_{4 r}(\psi(U)) \operatorname{sign}(c) i^{-\operatorname{sign}(c(d+a \pm 2))} \times \sum_{ \pm} \pm \frac{1}{2 i|c| \sqrt{|d+a \pm 2|}} \\
& \times \sum_{\beta(\bmod c)} \sum_{\gamma=1}^{|d+a \pm 2|} \exp 2 \pi i r \frac{-c \gamma^{2}+(a-d) \gamma \beta+b \beta^{2}}{d+a \pm 2}
\end{aligned}
$$

This simplifies to give 
Theorem'4.1. Suppose $U \in S L(2, \mathbb{Z})$ satisfies $|\operatorname{Tr} U|>2$. Then the Witten invariant of the torus bundle $\Sigma_{U}$ in the canonical framing is given by

$$
\begin{aligned}
Z\left(\Sigma_{U}, r ; 0\right)= & e_{4 r}(\psi(U)) \operatorname{sign}(d+a \mp 2) \times \sum_{ \pm} \pm \frac{1}{2|c| \sqrt{|d+a \mp 2|}} \\
& \times \sum_{\beta(\bmod c)} \sum_{\gamma=1}^{|d+a \mp 2|} \exp 2 \pi i r \frac{-c \gamma^{2}+(a-d) \gamma \beta+b \beta^{2}}{d+a \mp 2} .
\end{aligned}
$$

\subsection{Torus Bundle Invariants for General $G$}

This section gives a similar calculation of the Chern-Simons-Witten invariant for the torus bundles $\Sigma_{U}$, where $U=T^{p} S$ in terms of the generators (2.1), for a general compact simply connected Lie group $G$ with Lie algebra $\mathfrak{g}$ and Cartan subalgebra t. For simplicity we shall assume $G$ is simply laced, i.e., all the roots of $G$ have the same length. First we need to list the values of the representation of $P S L(2, \mathbb{Z})$ corresponding to general $G$ : this requires considerable notation, which is collected in the Appendix (Sect. A.1).

Proposition 4.2. In terms of the basis for $\mathscr{H}_{\Sigma}^{(k)}$ given by the level $k$ weights (A.16), the representation of $S L(2, \mathbb{Z})$ is ([14] (A.44)):

$$
\begin{aligned}
S_{\lambda, \mu} & =\frac{i^{\mid \Delta_{+}}}{r^{l / 2}}\left|\frac{\operatorname{vol} \Lambda^{w}}{\operatorname{vol} \Lambda^{R}}\right|^{1 / 2} \sum_{w \in W} \operatorname{det}(w) \exp \left(-\frac{2 \pi i}{r}\langle w(\lambda+\varrho), \mu+\varrho\rangle\right), \\
T_{\lambda, \mu} & =\delta_{\lambda, \mu} \exp \left(\frac{i \pi}{r}\langle\lambda+\varrho, \lambda+\varrho\rangle-\frac{i \pi}{h}\langle\varrho, \varrho\rangle\right) \\
& =\delta_{\lambda \mu} \exp 2 \pi i\left(H_{\lambda}-c / 24\right) .
\end{aligned}
$$

Here $\lambda$ and $\mu$ satisfy (A.16), and $\left|\Delta_{+}\right|$is the number of positive roots. The conformal weight $H_{\lambda}$ and central charge $c$ are defined in Sect. A.2.5 see also [21], Proposition 13.7.

We also need a more general version of the reciprocity formula for Gauss sums, whose proof, like that of the one-dimensional reciprocity formula (2.4), is a verbatim generalization of an argument in [7]). Consider a real vector space $V$ of dimension $l$ with inner product $\langle$,$\rangle and a lattice \Lambda$ in $V$ with dual lattice $\Lambda^{*}$. We introduce an integer $r$, a self-adjoint automorphism $B: V \rightarrow V$, and an element $\psi$ of $V$. We assume

$$
\begin{array}{lll}
\frac{1}{2}\langle\lambda, B r \lambda\rangle, & \langle\lambda, B \eta\rangle, & r\langle\lambda, \psi\rangle \in \mathbb{Z} \forall \lambda, \eta \in \Lambda, \\
\frac{1}{2}\langle\mu, B r \mu\rangle, & \langle\mu, r \xi\rangle, & r\langle\mu, \psi\rangle \in \mathbb{Z} \forall \mu, \xi \in \Lambda^{*} .
\end{array}
$$

Under these assumptions we have

Proposition 4.3 (Reciprocity Formula for Gauss Sums).

$$
\begin{aligned}
& \operatorname{vol}\left(\Lambda^{*}\right) \sum_{\lambda \in \Lambda / r \Lambda} \exp i \pi\langle\lambda, B \lambda / r\rangle \exp 2 \pi i\langle\lambda, \psi\rangle \\
& =\left(\operatorname{det} \frac{B}{i}\right)^{-1 / 2} r^{l / 2} \sum_{\mu \in \Lambda^{*} / B \Lambda^{*}} \exp \left\{-i \pi\left\langle\mu+\psi, r B^{-1}(\mu+\psi)\right\rangle\right\} .
\end{aligned}
$$

\footnotetext{
5 We correct an error in the formula in [14]. Note that [14] writes this in terms of a lattice $M$ and its dual $M^{*}$ : for simply laced groups, the identification of $M$ with $\Lambda^{R}$ is given by [21], 6.5.8
} 
Now we can exhibit the formula for the torus bundle invariant for general $G$.

We refer to the notation of Sect. A.1 and A.2, where in particular the weight lattice $\Lambda^{w}$ and the coroot lattice $\Lambda^{R}$ are introduced, and general properties of roots and weights discussed. The basic inner product on $\mathfrak{t}$ is denoted $\langle, \cdot\rangle$. The conjugacy classes of $U \in S L(2, \mathbb{Z})$ for which $c=1$ can be represented by $U=T^{p} S$. Using formulas (4.9) and (4.10), we obtain

$$
\begin{aligned}
& Z\left(\Sigma_{U}, r ; \psi(U)\right) \\
& =\operatorname{Tr}\left(T^{p} S\right)=i^{\left|\Delta_{+}\right|} \frac{1}{r^{l / 2}}\left|\frac{\operatorname{vol} \Lambda^{w}}{\operatorname{vol} \Lambda^{R}}\right|^{1 / 2} \exp \left\{-\frac{p i \pi\langle\varrho, \varrho\rangle}{h}\right\} \\
& \quad \times \sum_{w \in W} \operatorname{det}(w) \sum_{\lambda} \exp \left\{\frac{i \pi}{r}\left\langle\left(p-w-w^{-1}\right)(\lambda+\varrho), \lambda+\varrho\right\rangle\right\},
\end{aligned}
$$

where the sum is over $\lambda \in \Lambda^{w}$ satisfying (A.16).

We shall later wish to compare this expression with the semiclassical approximation (Proposition 5.15). This requires us to recast (4.12) in a form where the parameter $r$ appears in the numerator of each exponential rather than in the denominator.

We must first alter the sum in (4.12) using some symmetries so we are summing over something of the form $\mathbb{Z}^{n} / N \mathbb{Z}^{n}$ for $N$ some integer. Then we must apply the reciprocity formula to convert this sum to a sum over points in a dual lattice. Let us analyse the symmetries of the trace sum (4.12) with a view to expressing it as a sum over $\Lambda^{w} / r \Lambda^{w}$. Define (for $\lambda \in \Lambda^{w}$ )

$$
g(\lambda)=\sum_{w \in W} \operatorname{det}(w) \cdot \exp \frac{\pi i\langle(p-2 \omega) \lambda, \lambda\rangle}{r}
$$

The trace is obtained by summing $g(\lambda)$ over weights

$$
\left\{\lambda=\mu+\varrho: \mu \in \overline{\mathrm{FWC}},\left\langle\mu, \alpha_{m}\right\rangle \leq k\right\}
$$

in other words we must compute the sum

$$
\sum_{\lambda} g(\lambda)
$$

where the sum is over $\left\{\lambda \in \mathrm{FWC} \mid\left\langle\lambda, \alpha_{m}\right\rangle<k+h\right\}$.

Proposition 4.4. $g(\lambda)$ is invariant under:

(i) $\lambda \rightarrow-\lambda$ (obvious.

(ii) $\lambda \rightarrow u \lambda, u \in W:$ for

$$
\langle u \lambda,(p-2 w) u \lambda\rangle=\left\langle\lambda,\left\{p-2\left(u^{-1} w u\right)\right\} \lambda\right\rangle .
$$

(iii) $\lambda \rightarrow \lambda+r h_{\alpha}$, $\alpha$ any root ( $h_{\alpha}$ denotes the corresponding coroot $2 \alpha /\langle\alpha, \alpha\rangle$.) For

$$
\begin{aligned}
\frac{1}{r} & \left\langle\lambda+r h_{\alpha},(p-2 w)\left(\lambda+r h_{\alpha}\right)\right\rangle \\
& =\frac{1}{r}\langle\lambda,(p-2 w) \lambda\rangle+2\left\langle h_{\alpha},(p-2 w) \lambda\right\rangle+r\left\langle h_{\alpha},(p-2 w) h_{\alpha}\right\rangle .
\end{aligned}
$$

The second term is obviously in $2 \mathbb{Z}$, since $h_{\alpha}$ is in the integer lattice. The third term is also in $2 \mathbb{Z}$, since $\left\langle h_{\alpha}, h_{\alpha}\right\rangle \in 2 \mathbb{Z}$ (a property of the basic inner product), and

$$
\left\langle h_{\alpha}, w h_{\alpha}\right\rangle=\frac{2\left\langle w \alpha, h_{\alpha}\right\rangle}{\langle\alpha, \alpha\rangle} .
$$


The highest root, a long root, is normalized to have length ${ }^{2}=2$ in the basic inner product, and the length squared of short roots is then $2 / n$ for some $n \in \mathbb{Z}$.

(iv) $g(\lambda)=0$ for a weight $\lambda$ with $\langle\lambda, \alpha\rangle=r n$ for any root $\alpha(n \in \mathbb{Z})$. For every even $w \in W$ there is an odd $w$ contributing the opposite amount to the sum $g(\lambda)$, namely $\tau w$ where $\tau$ is reflection in $\alpha$. For

$$
\tau \lambda=\lambda-r n h_{\alpha}
$$

so

$$
\langle\lambda, 2 \tau w \lambda\rangle-\langle\lambda, 2 w \lambda\rangle=-2 r n\left\langle h_{\alpha}, w \lambda\right\rangle \in 2 r \mathbb{Z}
$$

so

$$
\exp i \pi \frac{\langle p-2 \tau w \lambda, \lambda\rangle}{r}=\exp i \pi \frac{\langle p-2 w \lambda, \lambda\rangle}{r} .
$$

We refer to Sect. A.5 for general discussion of alcoves, and more generally $r$ alcoves. The trace formula (4.12) requires one to sum $g(\lambda)$ over weights $\lambda$ in the interior of one $r$-alcove (and (iv) above says that weights on the boundary of the alcove do not contribute.) By Proposition 4.4, all $r$-alcoves contribute the same amount to such a sum. They are permuted simply transitively ([32], 5.14) by $W \tilde{\times} r \Lambda^{R} \subset W_{\text {aff. }}$.

If $\mathscr{S}$ is the sum of $g(\lambda)$ over one $r$-alcove, and if $N$ is a positive integer such that $\Lambda^{w} / N r \Lambda^{w}$ is precisely tiled by $r$-alcoves, then the number of $r$-alcoves is obtained by dividing the volume of $\Lambda^{w} / N r \Lambda^{w}$ by the volume of one $r$-alcove, which is $r^{l} \operatorname{vol} \Lambda^{R} /|W|$. Thus we have

$$
\sum_{\lambda \in \Lambda^{w} / N r \Lambda^{w}} g(\lambda)=N^{l}|W| \operatorname{vol}\left(\Lambda^{w}\right) / \operatorname{vol}\left(\Lambda^{R}\right) \mathscr{S} .
$$

In other words, the trace sum (4.12) gives

$$
\begin{aligned}
Z\left(\Sigma_{U}, r ; \psi(U)\right)= & i^{\left|\Delta_{+}\right|}\left|\frac{\operatorname{vol}\left(\Lambda^{w}\right)}{r^{l} \operatorname{vol}\left(\Lambda^{R}\right)}\right|^{1 / 2} \frac{\operatorname{vol}\left(\Lambda^{R}\right)}{N^{l}|W| \operatorname{vol}\left(\Lambda^{w}\right)} \exp -\frac{p \pi i\langle\varrho, \varrho\rangle}{h} \\
& \times \sum_{w \in W} \operatorname{det}(w) \sum_{\lambda \in \Lambda^{w} / N r \Lambda^{w}} \exp \frac{\pi i\left\langle\left(p-w-w^{-1}\right) \lambda, \lambda\right\rangle}{r} .
\end{aligned}
$$

The lattice volume factors in this sum combine to give $\operatorname{vol} \Lambda^{R}$ (since $\operatorname{vol} \Lambda^{w}=$ $\left.\left(\operatorname{vol} \Lambda^{R}\right)^{-1}\right)$.

Define

$$
B=p-w-w^{-1},
$$

an endomorphism of $t$. We wish to apply the reciprocity formula (Proposition 4.3) to relate

$$
\sum_{\mu \in \Lambda^{w} / r \Lambda^{w}} \exp \frac{i \pi\langle\mu, B \mu\rangle}{r}
$$

(which appears in (4.12)) to

$$
\sum_{\lambda \in \Lambda^{R} / B \Lambda^{R}} \exp -i \pi r\left\langle\lambda, B^{-1} \lambda\right\rangle
$$


(which will appear in the semiclassical calculation of Proposition 5.15). We cannot do this as stated, for the conditions (4.11) are not satisfied. However, we can choose $N \in \mathbb{Z}$ so that they are satisfied if we replace $r$ and $B$ by $r N, B N$. This gives

$$
\begin{aligned}
& \operatorname{vol}\left(\Lambda^{R}\right) \sum_{\lambda \in \Lambda^{w} / r N \Lambda^{w}} \exp \frac{i \pi\langle B \lambda, \lambda\rangle}{r} \\
& =\operatorname{det}(B / i)^{-1 / 2} r^{l / 2} N^{l} \times \sum_{\mu \in \Lambda^{R} / B \Lambda^{R}} \exp -i \pi\left\langle\mu, r B^{-1} \mu\right\rangle,
\end{aligned}
$$

by the reciprocity formula (4.3).

Using this, the trace sum (4.13) becomes

$$
\begin{aligned}
Z\left(\Sigma_{U}, r ; \psi(U)\right)= & \operatorname{Tr} \mathscr{B}(U)=\exp \left\{-\frac{p \pi i\langle\varrho, \varrho\rangle}{h}\right\} i^{\left|\Delta_{+}\right|} \exp \left\{\frac{i \pi l \operatorname{sgn} \operatorname{det}(B)}{4}\right\} \\
& \times \frac{|\operatorname{det}(B)|^{-1 / 2}}{|W|} \sum_{w \in W} \operatorname{det}(w) \sum_{\mu \in \Lambda^{R} / B \Lambda^{R}} \exp -i \pi\left\langle\mu, r B^{-1} \mu\right\rangle .
\end{aligned}
$$

All the lattice volume factors have cancelled out.

Formula (4.15) is not in the canonical framing. If we correct it using the framing $\psi(U)=-p+3 \operatorname{sign}(p)$ from (4.4) and

$$
e^{2 \pi \imath c / 24}=\exp \left\{2 \pi i \frac{\langle\varrho, \varrho\rangle}{2 h}\left(\frac{r-h}{r}\right)\right\}
$$

(see Sect. 2.4), we obtain the formula in the canonical framing:

$$
\begin{aligned}
Z\left(\Sigma_{U}, r ; 0\right)= & \exp \left\{-i \pi\langle\varrho, \varrho\rangle \frac{(p-3 \operatorname{sign}(p))}{r}\right\} \\
& \times \exp \left\{-3 \pi i \frac{\langle\varrho, \varrho\rangle}{h}\right\} i^{\left|\Delta_{+}\right|} \sum_{w \in W} \operatorname{det}(w) \\
& \times \exp \left\{\frac{i \pi l \operatorname{sgn} \operatorname{det}(B)}{4}\right\} \frac{|\operatorname{det}(B)|^{-1 / 2}}{|W|} \sum_{\mu \in \Lambda^{R} / B \Lambda^{R}} \\
& \times \exp -i \pi\left\langle\mu, r B^{-1} \mu\right\rangle .
\end{aligned}
$$

After some simplification this becomes

Proposition 4.5. If $U=T^{p} S$ and $|p|>2$, the Witten invariant of the torus bundle $\Sigma_{U}$ for an arbitrary simply laced compact connected simply connected group $G$ in the canonical framing is given by

$$
\begin{aligned}
Z\left(\Sigma_{U}, r ; 0\right)= & \exp \left\{-i \pi\langle\varrho, \varrho\rangle \frac{(p-3 \operatorname{sign}(p))}{r}\right\}(\operatorname{sign} p)^{l} \sum_{w \in W} \operatorname{det}(w) \\
& \times \frac{|\operatorname{det}(B)|^{-1 / 2}}{|W|} \sum_{\mu \in \Lambda^{R} / B \Lambda^{R}} \exp -i \pi\left\langle\mu, r B^{-1} \mu\right\rangle
\end{aligned}
$$




\section{Formula for the Large $r$ Limit}

In this section, we compare our results for the CSW invariants of lens spaces and torus bundles (Sect. 3.4) with the path integral formulas for the asymptotic behaviour of the Chern-Simons partition function as $r \rightarrow \infty$. This path integral prediction was originally obtained by Witten [40]. A more refined version of the path integral prediction is given in [11], (1.36). One has

$$
\begin{aligned}
Z(M, r) \sim & Z_{\mathrm{sc}}(M, r) \\
= & \frac{1}{|Z(G)|} e^{-i \pi(\operatorname{dim} G)\left(1+b^{1}(M)\right) / 4} \sum_{A} e^{2 \pi i r \operatorname{CS}(A)} v\left[\tau_{0}(M, A)^{1 / 2}\right] \\
& \times \tau_{1}(M, A)^{1 / 2} e^{-2 \pi i\left(I_{A} / 4+\left(\operatorname{dim} H_{A}^{0}+\operatorname{dim} H_{A}^{1}\right) / 8\right)} r^{\left(\operatorname{dim} H_{A}^{1}-\operatorname{dim} H_{A}^{0}\right) / 2} .
\end{aligned}
$$

Here, we sum over the gauge equivalence classes of flat connections $A$. The notation is as follows. We denote the centre of $G$ by $Z(G)$, and the first Betti number of $M$ by $b^{1}(M)$. The element $\operatorname{CS}(A)$ is the Chern-Simons invariant of the flat connection $A$, given by (1.2). For a definition of the Reidemeister-Ray-Singer torsion, we refer to $[12,19$ or 35]. The square root of the torsion associated to the flat connection $A$ is denoted by $\tau(M, A)^{1 / 2}$ : it is canonically an element $\tau_{0}(M, A)^{1 / 2} \otimes \tau_{1}(M, A)^{1 / 2}$ of $\Lambda^{\mathrm{max}} H^{0}\left(M, d_{A}\right) \otimes\left\{\Lambda^{\mathrm{max}} H^{1}\left(M, d_{A}\right)\right\}^{*}$. We denote by $v$ a volume on $\Lambda^{\mathrm{max}} H^{0}\left(M, d_{A}\right)$, so that $v\left[\tau_{0}(M, A)^{1 / 2}\right] \in \mathbb{C}$. In other words, once one chooses an element $v$ of $\Lambda^{\max } H^{0}\left(M, d_{A}\right)^{*}$, the square root of the torsion is a volume on $H^{1}\left(M, d_{A}\right)$, which is the Zariski tangent space of the moduli space of gauge equivalence classes of flat connections on $M$. A canonical such element $v \in \Lambda^{\max } H^{0}\left(M ; d_{A}\right)^{*}$ is obtained from the basic inner product (Sect. A.1) on $H^{0}\left(M ; d_{A}\right) \subset \mathfrak{g} .^{6}$

The integer $I_{A}$ is the spectral flow of the family of operators

$$
D_{A}=\left[\begin{array}{cc}
* d_{A} & -d_{A} * \\
d_{A} & 0
\end{array}\right]
$$

on $\Omega^{1}(M, \mathfrak{g}) \otimes \Omega^{3}(M, \mathfrak{g})$, where $A=A(t)$ is a path of connections running from the product connection $A_{0}$ to the flat connection $A$. We denote $H^{i}\left(M, d_{A}\right)$ by $H_{A}^{i}$.

Formula (5.1) was stated in [11] only for the case $G=S U(2), \operatorname{dim} H^{0}\left(M, d_{A}\right) \neq$ $0, \operatorname{dim} H^{1}\left(M, d_{A}\right)=0$; however, it extends also to the case when $\operatorname{dim} H^{1}\left(M, d_{A}\right) \neq 0$, by interpreting $\tau(M, A)^{1 / 2}$ as a volume on the space of gauge equivalence classes of flat connections on $M$ and the sum as an integral over that space. ${ }^{7}$

In this section, we explicitly demonstrate agreement of these results with the path integral asymptotic expansion for $Z(M, r)$ as $r \rightarrow \infty$, which was described in [11] and [40]. The values of the Chern-Simons invariants of flat connections on the torus bundle $\Sigma_{U}$ are given by a certain cocycle ([5], Sect.4.4.3) for the action of the automorphism group $\operatorname{Aut}(P)$ of a bundle $P$ over $\Sigma$ on the space of connections $\mathscr{A}(\Sigma)$ : below (Theorem 5.11) we obtain values for $\operatorname{CS}(A)$ explicitly when $\Sigma$ is a torus, through an alternative interpretation of this cocycle. Values for the Reidemeister torsion of flat connections on $\Sigma_{U}$ will be derived in a companion paper [18]. For the

\footnotetext{
${ }^{6}$ Although this choice of $v$ gives the correct normalization for the limit $Z(L(p, q), r)$ as $r \rightarrow \infty$, we do not obtain the correct normalization for $Z\left(S^{3}, r\right)=\mathscr{R}(S)_{11} \sim \sqrt{2} \pi r^{-3 / 2}$ : we have no explanation for the factor $\pi$ that appears here

7 This is true provided the Zariski tangent space $H^{1}\left(M, d_{A}\right)$ equals the actual tangent space to the moduli space of gauge equivalence classes of flat connections on $M$
} 
torus bundles, we have not been able to compute the spectral flows, but we conjecture a value based on the TQFT formulas for $Z\left(\Sigma_{U}, r\right)$.

Sect. 5.1 exhibits the precise agreement for the case of lens spaces and $G=S U(2)$. In preparation for the treatment of torus bundles, Sect. 5.2 describes results from [18] on the terms appearing in the asymptotic expansion for mapping tori (i.e., ChernSimons invariants and Reidemeister torsion of flat connections). Our results on torus bundles are given in Sect. 5.3. In contrast to our lens space results, which are complete, the torus bundle results have some details unresolved: these are listed in Remark 5.17. Nonetheless, in the torus bundle case almost all of the quantities appearing in the TQFT formula for the Witten invariant may be identified with corresponding quantities appearing in the semiclassical formula.

Remark 5.1. For the families of three-manifolds we have treated, the mechanism behind the proof that $Z(M, r)$ satisfies the asymptotic expansion is the reciprocity formula for Gauss sums (2.4), (4.3). This formula relies on the Fourier transform (via the Poisson summation formula): the Fourier transform is essentially what transforms the TQFT formula for the Witten invariant (where $r$ appears in the denominator of exponentials, as in the formula (2.3) for the representation of $\operatorname{PSL}(2, \mathbb{Z})$ ) to the semiclassical formula (5.1) where $r$ appears multiplying $\operatorname{CS}(A)$ in the numerator of exponentials.

Since the semiclassical formula (5.1) is expected to hold for all three-manifolds, we expect that the Witten invariants will quite generally satisfy some number-theoretic identities that facilitate such a transformation. Moreover, we speculate that the Fourier transform may be the basic mechanism behind these identities. Several authors have already studied the number theoretic properties of partition functions defined using quantum groups [29]: it is a challenge to extend the understanding of the link between quantum groups and number theory to find a number theoretic mechanism behind the asymptotic expansion.

\subsection{Lens Spaces}

The fundamental group $\pi_{1}$ of $L(p, q)$ is $\mathbb{Z}_{p}$, so representations $\varrho$ of $\pi_{1}$ in $S U(2)$ are indexed by $n=0, \ldots, p-1$ in the obvious way (i.e., $\varrho_{n}$ sends the generator to $\left.e^{2 \pi i n / p}\right)$, and $\varrho_{n}$ and $\varrho_{p-n}$ are conjugate. The Chern-Simons invariants $\operatorname{CS}\left(\varrho_{n}\right)$ and torsion $\tau\left(L(p, q), \varrho_{n}\right)$ of these flat connections have been calculated $[10,11,26]$ : they are $([11](2.20),(2.25))$

$$
\begin{aligned}
\operatorname{CS}\left(\varrho_{n}\right) & =q^{*} n^{2} / p \\
v\left[\tau\left(L(p, q), \varrho_{n}\right)^{1 / 2}\right] & =\frac{4 \sqrt{2}}{\sqrt{p}}\left|\sin \frac{2 \pi n}{p} \sin \frac{2 \pi q^{*} n}{p}\right|,
\end{aligned}
$$

where $q^{*} q=1(\bmod p) .^{8}$

Finally, the spectral flow is given by the following proposition. We are indebted to D.Zagier for its proof, which we include for completeness.

8 The factor $\sqrt{2}$ in the torsion does not appear in [11]; it arises from the volume element $v$ coming from the basic inner product, because the root of $S U(2)$ has length $\sqrt{2}$, whereas in [11] its length had implicitly been normalized to 1 
Proposition 5.2. The spectral flow $I_{n}$ from the product connection $A_{0}$ to $A_{n}$ is given by

$$
(-i)^{I_{n}}=(-i) \operatorname{sign}\left(\sin \frac{2 \pi q^{*} n}{p} \sin \frac{2 \pi n}{p}\right)
$$

i.e.,

$$
I_{n}(\bmod 4)= \begin{cases}1, & q^{*} n<\frac{p}{2} \\ -1, & q^{*} n>\frac{p}{2} .\end{cases}
$$

Proof. We have from [11] (2.21):

$$
\begin{aligned}
I_{n}+2 & =\frac{8 q^{*} n^{2}}{p}+\frac{2}{p} \sum_{k=1}^{p-1} \cot \frac{\pi k}{p} \cot \frac{\pi q k}{p} \sin ^{2} \frac{2 \pi n k}{p}, \\
& =\frac{8 q^{*} n^{2}}{p}+\frac{1}{2 p} \sum_{\substack{\lambda^{p}=1 \\
\lambda \neq 1}} \frac{\lambda+1}{\lambda-1} \frac{\lambda^{q}+1}{\lambda^{q}-1}\left(\lambda^{n}-\lambda^{-n}\right)^{2} .
\end{aligned}
$$

We make the expansion

$$
\begin{aligned}
\left(\lambda^{n}-\lambda^{-n}\right) \frac{\lambda+1}{\lambda-1} & =(\lambda+1)\left(\lambda^{n-1}+\lambda^{n-2}+\ldots+\lambda^{-n}\right) \\
& =\lambda^{n}+2 \lambda^{n-1}+\ldots+2 \lambda^{-n+1}+\lambda^{-n}
\end{aligned}
$$

Similarly, we rewrite the second factor $\lambda^{n}-\lambda^{-n}$ as $\left(\lambda^{q}\right)^{q^{*} n}-\left(\lambda^{q}\right)^{-q^{*} n}$, so we get

$$
\left(\lambda^{n}-\lambda^{-n}\right) \frac{\lambda^{q}+1}{\lambda^{q}-1}=\left(\lambda^{q}\right)^{q^{*} n}+2\left(\lambda^{q}\right)^{q^{*} n-1}+\ldots+2\left(\lambda^{q}\right)^{-q^{*} n+1}+\left(\lambda^{q}\right)^{-q^{*} n} .
$$

So

$$
\begin{aligned}
I_{n}+2= & \frac{1}{2 p} \sum_{\lambda^{p}=1}\left(\lambda^{n}+2 \lambda^{n-1}+\ldots+2 \lambda^{-n+1}+\lambda^{-n}\right) \\
& \times\left(\left(\lambda^{q}\right)^{q^{*} n}+2\left(\lambda^{q}\right)^{q^{*} n-1}+\ldots+2\left(\lambda^{q}\right)^{-q^{*} n+1}+\left(\lambda^{q}\right)^{-q^{*} n}\right) .
\end{aligned}
$$

(One verifies that the contribution to this sum from $\lambda=1$ is in fact the term $\frac{8 q^{*} n^{2}}{p}$ in (5.5).) In other words,

$$
\begin{aligned}
I_{n}+2= & \frac{1}{p} \sum_{\lambda^{p}=1}\left(\lambda^{n}+2 \lambda^{n-1}+\ldots+2 \lambda^{-n+1}+\lambda^{-n}\right) \\
& \times\left(1+2 \lambda^{q}+\ldots+2\left(\lambda^{q}\right)^{n q^{*}-1}+\lambda^{n}\right) .
\end{aligned}
$$

Since

$$
\sum_{\lambda^{p}=1} \lambda^{r}= \begin{cases}p, & p \mid r \\ 0, & \text { otherwise }\end{cases}
$$

we have $(\bmod 4)$

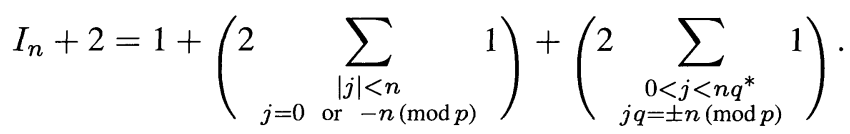


In other words

$$
I_{n}=1+2 \sum_{\substack{0<j<n q^{*} \\ j q= \pm n(\bmod p)}} 1(\bmod 4)
$$

We claim the sum is even if $q^{*} n<\frac{p}{2}$, and odd otherwise. This is true because the number of $j= \pm q^{*} n(\bmod p)$ in an interval $[k p,(k+1) p]$ is 2 , so since $n q^{*}$ is the first value of $j$ not contributing to our sum (5.6), the interval $[k p,(k+1) p]$ containing $n q^{*}$ contributes 0 if $n q^{*}<\frac{p}{2}$ and 1 otherwise. This completes the proof.

We compare these values with the prediction of the path integral formula. In the limit $r \rightarrow \infty$, formula (3.9) gives

$$
Z(L(p, q), r ; 0) \simeq i \sqrt{\frac{2}{r p}} \sum_{n=1}^{p} \exp \frac{2 \pi i q^{*} r n^{2}}{p} \sin \frac{2 \pi q^{*} n}{p} \sin \frac{2 \pi n}{p} .
$$

This agrees precisely with the path integral prediction (5.1).

Remark 5.3. Notice that the semiclassical formula (5.7) does not agree with the exact formula for $Z(L, p, q), r ; 0)$ given by Theorem 3.4, but only with its $r \rightarrow \infty$ limit: in other words, stationary phase is not exact.

\subsection{Mapping Tori}

In this section, $\Sigma$ is a surface of arbitrary genus, and $\beta: \Sigma \rightarrow \Sigma$ a diffeomorphism. The mapping torus $\Sigma_{\beta}$ with fibre $\Sigma$ and monodromy $\beta$ was defined by (4.1). The Witten invariants of these 3-manifolds are treated more extensively in a companion paper [18], in relation to a $(0+1)$ dimensional analogue of the Chern-Simons path integral (symplectic quantum mechanics) based on Floer's symplectic action functional. In that paper, we identify the semiclassical approximation of a special case of the $(0+1)$ dimensional field theory with the semiclassical approximation (5.1) to the ChernSimons path integral for the mapping torus $\Sigma_{\beta}$. To this end, [18] treats the ChernSimons invariants and Reidemeister torsion of flat connections on mapping tori. In this section, we summarize from [18] those results we shall need for the explicit treatment of mapping tori of tori.

\subsubsection{Preliminaries. Let $\Sigma$ be a surface. We recall the following:}

1. The surface $\Sigma$ is assumed equipped with the (topologically trivial) principal $G$ bundle $P$, and we assume a lift $\widetilde{\beta}$ of $\beta$ to $P$ has been chosen. One may then define a mapping torus bundle $P_{\tilde{\beta}} \rightarrow \Sigma_{\beta}$, as the mapping torus of $P$ under $\tilde{\beta}$. For reference, we specify a flat connection $A_{0}$ on $\Sigma$ such that $\tilde{\beta}^{*} A_{0}=A_{0}$. (For instance, one may do this by picking a trivialization of $P$, and taking $\beta$ to be the corresponding trivial lift of $\beta$ and $A_{0}$ to be the product connection.)

2. We denote by $\mathscr{b}(\Sigma)$ the space of all connections on $P \rightarrow \Sigma$, and by $\mathscr{C}(\Sigma)$ the moduli space of gauge equivalence classes of flat connections on $P$. This space of course has an equivalent description as the moduli space of conjugacy classes of representations of $\pi_{1} \Sigma$ in $G$. This moduli space is equipped with the basic symplectic form

$$
\omega(a, b)=\frac{1}{2 \pi} \int_{\Sigma} \operatorname{Tr}(a \wedge b)
$$


for $a, b \in T_{A} \mathscr{C}(\Sigma)=H^{1}\left(\Sigma, d_{A}\right)$. There is a prequantum line bundle with connection $(\mathscr{B}, \theta)$ over $\mathscr{C}(\Sigma)$; this is by definition a line bundle $\mathscr{L}$ over $\mathscr{l}(\Sigma)$ equipped with a connection $\theta$ whose curvature is $-i \omega$.

3. The bundle map $\tilde{\beta}: P \rightarrow P$ covering $\beta$ induces a diffeomorphism $f_{\beta}: \mathscr{M b}(\Sigma) \rightarrow$ $\mathscr{O}(\Sigma)$. This has a lift $\tilde{f}_{\beta}: \mathscr{L} \rightarrow \mathscr{L}$ preserving the connection $\theta$. Such a lift is unique up to multiplication by a constant $U(1)$ phase factor.

4. We denote by $\mathscr{C}\left(\Sigma_{\beta}\right)$ the moduli space of gauge equivalence classes of flat connections on the bundle $P_{\tilde{\beta}}$ over the mapping torus $\Sigma_{\beta}$.

First observe that a connection $\tilde{A}$ on $P_{\tilde{\beta}} \rightarrow \Sigma_{\beta}$ may be written as

$$
\tilde{A}=A(t)+\Phi(t) d t \quad(t \in \mathbb{R}),
$$

where $A(t) \in \mathscr{B}(\Sigma)$ and $\Phi(t) \in \Omega^{0}(\Sigma, \operatorname{ad}(P))$ satisfy the periodicity conditions

$$
\tilde{\beta}^{*} A(t+1)=A(t), \quad \tilde{\beta}^{*} \Phi(t+1)=\Phi(t) .
$$

The following facts are treated in more detail in [18]: see also [8], especially Appendix A. The treatment of the Chern-Simons functional given in Proposition 5.5 follows from [5], Sect. 4.4.3.

Lemma 5.4. There is a surjective map from the moduli space of gauge equivalence classes of flat connections $\tilde{A}=A(t)+\Phi(t) d t$ on $\Sigma_{\beta}$ to the set of fixed points of $f_{\beta}: \mathscr{M C}(\Sigma) \rightarrow \mathscr{M}(\Sigma)$, given by $[\tilde{A}] \rightarrow[A(0)]$.

Proposition 5.5. One may choose the lift $\tilde{f}_{\beta}: \mathscr{L} \rightarrow \mathscr{L}$ so that the Chern-Simons action $e^{2 \pi i \operatorname{CS}(A)}$ at a flat connection $\tilde{A}$ on $\Sigma_{\beta}$ is equal to $\operatorname{Tr} \tilde{f}_{\beta}$, the trace of $\tilde{f}_{\beta}$ on the fibre of the prequentum line bundle over the fixed point $[A(0)] \in \mathscr{M}(\Sigma)$.

5.2.2. Reidemeister Torsion. We refer also to the following results from [18]. The first Betti number is $b^{1}\left(\Sigma_{\beta}\right)=1$. The fundamental group $\pi_{1}\left(\Sigma_{\beta}\right)$ is $\mathbb{Z} \tilde{\times} \pi_{1}(\Sigma)$, where $\mathbb{Z}$ acts on $\pi_{1}(\Sigma)$ via the diffeomorphism $\beta$. The moduli space $\mathscr{l}\left(\Sigma_{\beta}\right)$ of conjugacy classes of representations of $\pi_{1}\left(\Sigma_{\beta}\right)$ in $G$ may thus be identified as

$$
\mathscr{M}\left(\Sigma_{\beta}\right)=\left\{\tilde{\varrho}=(\varrho, g) \mid \varrho \in \operatorname{Hom}\left(\pi_{1} \Sigma, G\right), g \in G, g \varrho g^{-1}=\beta^{*} \varrho\right\} / G .
$$

Denote by $\mathscr{C}_{\varrho}$ the subspace of $\mathscr{\mathscr { C }}\left(\Sigma_{\beta}\right)$ corresponding under the above identification to a particular element $\varrho \in \operatorname{Hom}\left(\pi_{1} \Sigma, G\right)$. One sees that if $(\varrho, g) \in \mathscr{L} b\left(\Sigma_{\beta}\right)$, then any $g$ is in the normalizer $N(\operatorname{Stab}(\varrho))$ of the stabilizer $\operatorname{Stab}(\varrho)$ of $\varrho$ under conjugation. Furthermore, if $(\varrho, g),\left(\varrho, g_{0}\right) \in \mathscr{M}_{\varrho}$, then $g^{-1} g_{0} \in \operatorname{Stab}(\varrho)$. Thus conjugation by $\operatorname{Stab}(\varrho)$ preserves the space $\operatorname{Stab}(\varrho) g$, and we may identify $\mathscr{C}_{\varrho}=\operatorname{Stab}(\varrho) g_{0} / \operatorname{Stab}(\varrho)$, where by this we mean the quotient of $\operatorname{Stab}(\varrho) g_{0}$ by the conjugation action of $\operatorname{Stab}(\varrho)$.

Let $\tilde{\varrho} \in \mathscr{C}_{\varrho}$, and let $\tilde{A}$ be a corresponding flat connection on $P_{\tilde{\beta}}$. Then the square root of the Reidemeister torsion $\tau\left(\Sigma_{\beta}, \tilde{A}\right)^{1 / 2}$ is an element of $\Lambda^{\max } H^{0}\left(\Sigma_{\beta}, d_{\tilde{A}}\right) \otimes$ $\Lambda^{\max } H^{1}\left(\Sigma_{\beta}, d_{\tilde{A}}\right)^{*}$. In other words, if we specify an element $v$ of $\Lambda^{\max } H^{0}\left(\Sigma_{\beta}, d_{\tilde{A}}\right)^{*}$,

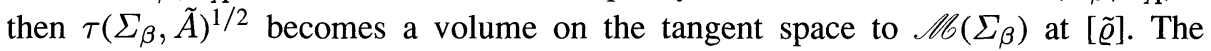
following proposition is proved in [18]:

Proposition 5.6. Assume that $f_{\beta}$ has isolated fixed points on $\mathscr{M b}(\Sigma)$. Then the following hold:

(a) For the mapping torus $\Sigma_{\beta}$, we have

$$
\operatorname{dim} H^{0}\left(\Sigma_{\beta}, d_{\tilde{A}}\right)=\operatorname{dim} H^{1}\left(\Sigma_{\beta}, d_{\tilde{A}}\right) .
$$


(b) For a suitable choice of the element $v$ of $\Lambda^{\max } H^{0}\left(\Sigma_{\beta}, d_{\tilde{A}}\right)^{*}$, the integral of the square root of the Reidemeister torsion over the subset $\mathscr{C}_{\varrho}$ of $\mathscr{M b}\left(\Sigma_{\beta}\right)$ is

$$
\int_{\mathscr{L}_{\varrho}} \tau\left(\Sigma_{\beta}, \tilde{A}\right)^{1 / 2}=\int_{(\varrho, g) \in \mathscr{H}_{\varrho}} \frac{1}{\left|\operatorname{det}\left(\operatorname{ad}(g) \tilde{\beta}^{*}-1\right)\right|^{1 / 2}} d \text { vol . }
$$

Here, $B=\operatorname{ad}(g) \tilde{\beta}^{*}-1 \in \operatorname{End}\left(H^{1}\left(\Sigma, d_{A}\right)\right)$, where $A$ is a flat connection on $P \rightarrow$ $\Sigma$ corresponding to the representation $\varrho$; we take the determinant of the restriction of $B$ to the orthocomplement of $\operatorname{Ker} B$. We take the volume element of $\mathscr{M}_{\varrho}=$ $\operatorname{Stab}(\varrho) g_{0} / \operatorname{Stab}(\varrho)$ in an appropriate metric.

\subsection{Torus Bundles over the Circle}

The case of mapping tori of $\Sigma$ when $\Sigma$ is a torus can be treated quite explicitly. Firstly, in this case there is a simple concrete description of the moduli space $\mathscr{C}(\Sigma)$ : it is the space $(T \times T) / W$, the product of two copies of the maximal torus quotiented by the diagonal action of the Weyl group. Secondly, there is an equally explicit description of the vector space $\mathscr{H}_{\Sigma}^{(k)}$ and the action of $S L(2, \mathbb{Z})$ on $\mathscr{H}_{\Sigma}^{(k)}$, as discussed in the Appendix.

Notation. The diffeomorphism $\beta$ of $\Sigma$ corresponds to an element $U \in S L(2, \mathbb{Z})$. We shall write $f_{U}$ for the corresponding map on $(T \times T) / W$, and $\tilde{f}_{U}$ for its lift to the prequantum line bundle $\mathscr{B}$ over $(T \times T / W$ or $T \times T$.

5.3.1. Semiclassical Formula for Torus Bundles. We shall define the contributions to the semiclassical formula starting by working on $T \times T$. Observe that if $A \in T \times T$ is a fixed point of a linear map $U$ acting on $(T \times T) / W$, then there is some $w \in W$ such that $A$ is a fixed point of $w U$ acting on $T \times T$. It is natural to consider all maps $f=w U$ on $T \times T$ and sum the contributions to the semiclassical formula corresponding to their fixed points. In fact, $w U A=A$ in $T \times T$ if and only if $w^{\prime} A$ solves $w^{\prime} w\left(w^{\prime}\right)^{-1} U\left(w^{\prime} A\right)=w^{\prime} A$, so it is necessary to divide our fixed point sum by $|W|$.

Remark 5.7. Reidemeister Torsion. Suppose $A$ is a fixed point of $w U$ in $T \times T$. The stabilizer $\operatorname{Stab}(A)$ of a generic point $A \in T \times T$ is the maximal torus $T$. Assume $\operatorname{Stab}(A)=T$. Then the quantity $\left|\operatorname{det}\left(1-\operatorname{ad}(g) \tilde{\beta}^{*}\right)\right|$ on $H^{1}(\Sigma, \operatorname{ad}(P))$ from Proposition 5.6 naturally corresponds to the quantity $\operatorname{det}(1-w U) .{ }^{9}$ The volume $\operatorname{vol}(\operatorname{Stab}(\varrho) g / \operatorname{Stab}(\varrho))$ may be identified with $\operatorname{vol}(\operatorname{Stab}(\varrho) \cap Z(g))=\operatorname{vol}(Z(w))$, the volume of the fixed point set of the element $w \in W$ corresponding to $g$. When $G=S U(2)$, the factor $\operatorname{vol}(\operatorname{Stab}(\varrho) \cap Z(g))$ is 2 for all $(\varrho, g)$, provided we assume we have a metric for which $\operatorname{vol}(T / W)=1 .^{10}$ This factor cancels the factor $|Z(G)|^{-1}=1 / 2$ in the semiclassical formula (5.1). We have not determined the factor $\operatorname{vol}(\operatorname{Stab}(\varrho) \cap Z(g))$ for a general group $G$.

\footnotetext{
9 We have not obtained this identification when $A$ is stabilized by a larger group that $T$. The formulas given below (Proposition 5.12, Proposition 5.15) assume the same formula for the torsion holds for such points $A$; this assumption appears to agree with the TQFT formulas for the Witten invariant

10 We remark that this is not the same as the normalization given by the basic inner product; we have no explanation for this discrepancy
} 
This prescription determines $\left.\operatorname{Tr} \tilde{f}_{w U}\right|_{A}$ and $\operatorname{det}\left(1-\operatorname{ad}(g) \tilde{\beta}^{*}\right)$ for $A \in T \times T$, provided $A$ is fixed by only one of the maps $w U$, i.e., provided no nontrivial element of $W$ fixes $A$. For those elements $A$ which are fixed by some element in $W$, we sum the contributions to the semiclassical formula for all $w U$ that fix $A$.

We now wish to compare with the formulas in Sect.4. We have not been able to obtain a prescription for the spectral flow, but make the following conjecture based on the TQFT formulas (4.8), (4.16) for the Witten invariant:

Conjecture 5.8. We have

$$
\begin{gathered}
e^{-i \pi I_{A} / 2} e^{-i \pi\left(\operatorname{dim} H_{A}^{1}+\operatorname{dim} H_{A}^{0}\right) / 4} e^{-i \pi \operatorname{dim} G\left(1+b^{1}\left(\Sigma_{U}\right)\right) / 4} \\
=(-i)^{I_{A}+\operatorname{dim} H_{A}^{1}+\operatorname{dim} G}=\operatorname{det}(w) \operatorname{sign}(\operatorname{Tr} U)^{l}
\end{gathered}
$$

where $w$ is an element of the Weyl group such that $w U$ fixes the point $A \in T \times T{ }^{11}$

The semiclassical formula is then, using Proposition 5.5, Proposition 5.6 and Conjecture 5.8:

$$
\begin{aligned}
Z_{\mathrm{sc}}\left(\Sigma_{U}, r\right)= & \frac{\operatorname{sign}(\operatorname{Tr} U)^{l}}{|W|} \sum_{w \in W} \sum_{\substack{A \in T \times T \\
w U A=A}} \operatorname{det} w \frac{\left(\left.\operatorname{Tr} \tilde{f}_{w U}\right|_{A}\right)^{r}}{|\operatorname{det}(1-w U)|^{1 / 2}} \\
& \times \frac{\operatorname{vol}(Z(w))}{|Z(G)|},
\end{aligned}
$$

where $Z(w)$ is the subgroup of $T$ that is fixed by the action of $w$. (As discussed above, for $G=S U(2)$, $\operatorname{vol} Z(w)=2$ for all $w$.)

We now discuss the lifting of $\beta: \Sigma \rightarrow \Sigma$ when $\Sigma$ is a torus: we refer to Sect. A.3, where the prequantum line bundle $\mathscr{L}$ is constructed. We may view $\mathfrak{t} \oplus \mathfrak{t}$ as a subspace $\mathbb{A}$ of the space of connections $\mathscr{C}$ on $\Sigma$, and the actions of $W$ and $\Lambda=\Lambda^{R} \oplus \Lambda^{R}$ on $\mathbb{A}$ as the restrictions to $\mathbb{A}$ of certain gauge transformations. The lifting of these actions to the prequantum line bundle is described in Sect. A.3; it is easy to check by explicit calculation that this lifting coincides with the lifting of the gauge group action via the Chern-Simons cocycle (see, for instance, [34]).

We need to choose a lift of $\beta=U: \Sigma \rightarrow \Sigma$ to $\tilde{U}: P \rightarrow P$, and a flat connection $A_{0}$ preserved by $\tilde{U}$. We do this by choosing a trivialization of $P$ and letting $A_{0}$ be the product connection and $\tilde{U}$ the trivial lift. This choice of $\tilde{U}$ then preserves the subspace $\mathbb{A} \subset \mathscr{A}$. We identify $A_{0}$ with $0 \in \mathfrak{t}$; this enables us to lift the action of $f_{U}$ on $(T \times T) / W$ to the linear action of $U \in S L(2, \mathbb{Z})$ on $\mathbb{A}$. Of course the connection on the symplectic affine space $\mathbb{A}$ is given by Lemma A.1: it is simply the restriction to $\mathbb{A}$ of the standard connection (A.8) on the space $\mathscr{A}$ of connections, whose curvature is $-i \omega$ for the symplectic form $\omega$ given by (A.7).

We now choose a lift of $f_{U}:(T \times T) / W \rightarrow(T \times T) / W$ to $\tilde{f}_{U}: \mathscr{B} \rightarrow \mathscr{L}$, preserving the connection. We choose the trivial lift to the trivial bundle over $\mathbb{A}$ :

$$
\tilde{f}_{U}(A, z)=(U A, z) .
$$

The detailed proof that the lifting (5.12) descends to give a lift to the prequantum line bundle over $(T \times T) / W$ is given in Sect. A. 4 .

Lemma 5.9. The lift (5.12) coincides precisely with the lift described in Proposition 5.5 .

11 This requires interpretation when there are more than one $w U$ fixing $A$ : see (1) of Remark 5.17 
Proof. The lift (5.12) is easily shown to preserve the connection (A.8) on $\mathscr{B}$. However, all lifts to the prequantum line bundle preserving the connection coincide up to a constant in $U(1)$. That this constant is 1 follows from the fact that the two lifts agree on the product connection $A_{0}$.

We now derive explicit formulas for the trace of $\tilde{f}_{U}$ at a fixed point. We refer to section A.3 of the Appendix. If $A$ is a fixed point of $f_{U}$ on $(T \times T) / W$, there are $w \in W$ and $\lambda \in \Lambda=\Lambda^{R} \otimes \Lambda^{R}$ such that

$$
w U A-A=\lambda
$$

in $\mathfrak{t} \oplus \mathfrak{t}$. The trace of $\tilde{f}_{U}=\tilde{f}_{w U}$ at a fixed point is computed as follows:

$$
\begin{aligned}
\tilde{f}_{w U}(A, v) & =(w U(A), v)=(A+\lambda, v) \\
& =\left(A, e_{\lambda}(A)^{-1} v\right),
\end{aligned}
$$

the last step using (A.9). In other words

$$
\text { Trace } \begin{aligned}
\left.\tilde{f}_{w U}\right|_{A} & =e_{\lambda}(A)^{-1} \\
& =\exp \frac{i}{2} \omega(A, \lambda) \varepsilon(\lambda) \quad \text { by (A.10). }
\end{aligned}
$$

Explicitly,

Lemma 5.10. The fixed points $A^{(\lambda)}$ of $f_{U}$ on $(T \times T) / W$ are in correspondence with $\lambda=\left(\lambda_{1}, \lambda_{2}\right) \in \Lambda /(w U-1) \Lambda$ : we define

$$
A^{(\lambda)}=\left[\begin{array}{l}
A_{1} \\
A_{2}
\end{array}\right]=(w U-1)^{-1} \lambda \in \mathfrak{t} \oplus \mathfrak{t}
$$

Furthermore, the trace of the lift $\tilde{f}_{w U}$ at the fixed point $A^{(\lambda)}$ is given by

$$
\text { Trace } \begin{aligned}
\left.\tilde{f}_{w U}\right|_{A^{(\lambda)}} & =\exp \frac{i}{2} \omega\left((w U-1)^{-1} \lambda, \lambda\right) \varepsilon(\lambda), \\
& =\exp -i \pi\left\langle(w U-1)^{-1} \lambda, S \lambda\right\rangle \varepsilon(\lambda) \quad \text { by (A.7), }
\end{aligned}
$$

where the theta-characteristic $\varepsilon(\lambda)$ is defined by (A.17).

By Proposition 5.9, we actually have that

$$
\text { Trace }\left.\tilde{f}_{w U}\right|_{A^{(\lambda)}}=\exp \left(2 \pi i \operatorname{CS}\left(\tilde{A}^{(\lambda)}\right)\right)
$$

where $\tilde{A}^{(\lambda)}$ is the flat connection on $\Sigma_{\beta}$ corresponding to $A^{(\lambda)}$. This proves:

Theorem 5.11. The Chern-Simons invariant of the flat connection $\tilde{A}^{(\lambda)}$ on the torus bundle $\Sigma_{U}$ is:

$$
\operatorname{CS}\left(\tilde{A}^{(\lambda)}\right)=\frac{1}{4 \pi} \omega\left((w U-1)^{-1} \lambda, \lambda\right)+ \begin{cases}0, & \varepsilon(\lambda)=1 \\ \frac{1}{2} & \varepsilon(\lambda)=-1 .\end{cases}
$$

Kirk and Klassen ([26], Theorem 5.6) have obtained this result for $G=S U(2)$.

5.3.2. $G=S U(2)$. We shall present the calculation of the semiclassical limit $Z_{\text {sc }}\left(\Sigma_{U}, r\right)$ (5.1) for the Chern-Simons partition function of the torus bundle $\Sigma_{U}$ for $\Sigma$ a torus. We shall see that the result agrees with the large $r$ limit (5.20) of the formula for $Z\left(\Sigma_{U}, r ; 0\right)$. 
We denote by $U$ an arbitrary element

$$
U=\left[\begin{array}{ll}
a & b \\
c & d
\end{array}\right] \in S L(2, \mathbb{Z})
$$

(provided $U$ is not parabolic, i.e., $\operatorname{Tr} U \neq \pm 2$.) We wish to compute the semiclassical formula (5.11) for the torus bundle partition function. We need the quantity

$$
\operatorname{det}(w U-1)=2 \mp(a+d) .
$$

Equation (A.7) and Theorem 5.11 allow us to evaluate the action: recalling that the theta-characteristic $\varepsilon(\lambda)$ is 1 for $G=S U(2)$, we have

$$
\begin{aligned}
(w U-1)^{-1} & =-\frac{1}{a+d \mp 2}\left[\begin{array}{cc}
d \mp 1 & -b \\
-c & a \mp 1
\end{array}\right], \\
\operatorname{CS}\left(\tilde{A}^{(\lambda)}\right) & =\frac{1}{2}\left\langle(w U-1)^{-1} \lambda, S \lambda\right\rangle \\
& =-\frac{1}{2}\left\langle\lambda,\left(w U^{t}-1\right)^{-1} S \lambda\right\rangle
\end{aligned}
$$

where

$$
S=\left[\begin{array}{cc}
0 & -1 \\
1 & 0
\end{array}\right] \in S L(2, \mathbb{Z})
$$

(Here, $\langle\cdot, \cdot\rangle$ is the inner product on $\mathfrak{t} \oplus \mathfrak{t}$, and $S \in S L(2, \mathbb{Z})$ acts on $\mathfrak{t} \oplus \mathfrak{t}$.) This becomes

$$
\operatorname{CS}\left(\tilde{A}^{(\lambda)}\right)=\frac{1}{a+d \mp 2}\left(-c \lambda_{1}^{2}+b \lambda_{2}^{2}+(a-d) \lambda_{1} \lambda_{2}\right)
$$

where we have used the coroot basis and the basic inner product $\langle\cdot, \cdot\rangle$ to identify $\Lambda^{R}$ with $\mathbb{Z}$. Our expression (5.11) then gives

Proposition 5.12. The semiclassical approximation to the $S U(2)$ Witten invariant for the torus bundle $\Sigma_{U}$ is given by

$$
\begin{aligned}
Z_{\mathrm{sc}}\left(\Sigma_{U}, r\right)= & \frac{\operatorname{sign}(d+a)}{2} \sum_{ \pm} \sum_{\lambda} \pm \frac{1}{\sqrt{|a+d \mp 2|}} \\
& \times \exp \left\{2 \pi i \frac{r}{a+d \mp 2}\left(-c \lambda_{1}^{2}+b{\lambda_{2}}^{2}+(a-d) \lambda_{1} \lambda_{2}\right)\right\},
\end{aligned}
$$

where we sum over $\lambda=\left(\lambda_{1}, \lambda_{2}\right) \in \Lambda /( \pm U-1) \Lambda$.

Assume now for simplicity that $U$ is hyperbolic, i.e., $|\operatorname{Tr}(U)|>2$. Then the expression (5.20) is equal to the large $r$ limit of the expression (4.8) from the TQFT formula for $\operatorname{Tr} \mathscr{R}$. One may see this as follows. Note that the result (5.20) is expressed as a sum over a fundamental domain of $\Lambda$ under the action of $B=1 \pm U$. The equivalence of this with (4.8) is established by the following observations:

1. $\operatorname{det} B=2 \pm a \pm d$.

2. The sum

$$
\sum_{\beta=1}^{|c||d+a \pm 2|} \sum_{\gamma=1}^{|d+a \pm 2|} \exp 2 \pi i r \frac{-c \gamma^{2}+(a-d) \gamma \beta+b \beta^{2}}{d+a \pm 2}
$$

equals $\mid$ det $B \mid$ times the sum in (4.8). Indeed, if $(m, n) \in \Lambda \cong \mathbb{Z}^{2}$ is such that $\operatorname{det} B$ divides $m$ and $n$, then $(m, n)$ is in $B \Lambda$, so the points $(\beta, \gamma)=(0,|d+a \pm 2|)$, 
$(\beta, \gamma)=(|c||d+a \pm 2|, 0)$ are in $B \Lambda$. The rectangle spanned by these two vectors has volume $|\operatorname{det} B|^{2}|c|$. Hence the sum (5.21) covers precisely $|\operatorname{det} B||c|$ fundamental domains for the action of $B$ (as it covers an integer number, each of which contains $|\operatorname{det} B|$ points.)

5.3.3. General $G$. In the case of general $G$ we shall restrict ourselves for simplicity to one specific family, namely those $U \in S L(2, \mathbb{Z})$ for which $c=1$.

Notation. $p$ will denote $\operatorname{Tr}(U)$.

Lemma 5.13. We have

$$
|\operatorname{det}(w \otimes U-1)|=\left|\operatorname{det}\left(p-w-w^{-1}\right)\right| .
$$

Here, the first determinant is the determinant of an automorphism of $\mathfrak{t} \oplus \mathfrak{t}=\mathfrak{t} \otimes \mathbb{R}^{2}$, while the second is the determinant of an automorphism of $\mathfrak{t}$.

Proof. If the eigenvalues of $U$ are $\lambda, \lambda^{-1}$ and those of $w$ are $\mu$ then this breaks up as

$$
\begin{aligned}
\text { LHS } & =\left|\prod_{\mu}(\lambda \mu-1)\left(\lambda^{-1} \mu-1\right)\left(\lambda \mu^{-1}-1\right)\left(\lambda^{-1} \mu^{-1}-1\right)\right|^{1 / 2} \\
& =\left|\prod_{\mu}\left(\lambda^{1 / 2} \mu^{1 / 2}-\lambda^{-1 / 2} \mu^{-1 / 2}\right)\left(\lambda^{-1 / 2} \mu^{1 / 2}-\lambda^{1 / 2} \mu^{-1 / 2}\right)\right| \\
& =\left|\prod_{\mu}\left(\lambda+\lambda^{-1}-\mu-\mu^{-1}\right)\right|=\text { RHS } .
\end{aligned}
$$

Lemma 5.14. $A$ basis of representatives $\lambda$ for $\Lambda /(w U-1) \Lambda$ is given by

$$
\lambda=(\sigma, 0), \quad \sigma \in \Lambda^{R} /\left(p-w-w^{-1}\right) \Lambda^{R} .
$$

Proof. By Lemma 5.13, these sets have the same number of elements. Now

where

$$
(w U-1) \Lambda=\left(U-w^{-1}\right) \Lambda
$$

$$
U-w^{-1}=\left[\begin{array}{cc}
a-w^{-1} & b \\
c & d-w^{-1}
\end{array}\right]
$$

As $c=1$, there is clearly a basis of representatives of the form $(\sigma, 0)$ (since for every $\tau \in \Lambda^{R}$,

$$
\left(U-w^{-1}\right)\left[\begin{array}{l}
\tau \\
0
\end{array}\right]
$$

is an element of $\left(U-w^{-1}\right) \Lambda$ with the second coordinate equal to $\tau$, so the second coordinate may be subtracted off from any set of representatives.)

Also,

$$
\left[\begin{array}{cc}
a-w^{-1} & b \\
c & d-w^{-1}
\end{array}\right]\left[\begin{array}{c}
-\left(d-w^{-1}\right) \sigma \\
\sigma
\end{array}\right]=\left[\begin{array}{c}
\left(p-w^{-1}-w\right) w^{-1} \sigma \\
0
\end{array}\right]
$$

so for any $\sigma \in \Lambda^{R},\left(\left(p-w-w^{-1}\right) \sigma, 0\right) \in(w U-1) \Lambda$.

Remark. Since a set of representatives $\lambda \in \Lambda$ can be chosen in the way described in Lemma 5.10, with the second component $\lambda_{2}$ equal to zero, it is easy to see that the theta characteristics $\varepsilon(\lambda)$ defined by (A.17) for the representatives $\lambda$ are 1 , 
We need the factor

$$
\operatorname{CS}\left(\tilde{A}^{(\lambda)}\right)=-\frac{1}{2}\left\langle A^{(\lambda)}, S \lambda\right\rangle=-\frac{1}{2}\left\langle A_{2}, \sigma\right\rangle,
$$

where $(w U-1) A^{(\lambda)}=\lambda$. Explicitly,

$$
\left[\begin{array}{cc}
(a w-1) & b w \\
c w & (d w-1)
\end{array}\right]\left[\begin{array}{l}
A_{1} \\
A_{2}
\end{array}\right]=\left[\begin{array}{l}
\sigma \\
0
\end{array}\right]
$$

su substituting for $A_{1}$, we obtain

$$
A_{2}=\left(w+w^{-1}-d-a\right)^{-1} \sigma .
$$

Combining (5.22) and (5.23), we have

$$
\exp 2 \pi i r \operatorname{CS}\left(\tilde{A}^{(\lambda)}\right)=\left(\operatorname{Tr} \tilde{f}_{A^{(\lambda)}}\right)^{r}=\exp -i \pi r\left\langle\left(p-w-w^{-1}\right) \sigma, \sigma\right\rangle .
$$

Substituting this, Conjecture 5.8 and the result of Lemma 5.13 into (5.11), we have

Proposition 5.15. The semiclassical formula for the torus bundle partition function for an arbitrary compact connected simply laced Lie group $G$ and $U=T^{p} S$ is

$$
\begin{aligned}
Z_{\mathrm{sc}}\left(\Sigma_{U}, r\right)= & \frac{\operatorname{sign}(p)^{l}}{|W|} \sum_{w \in W} \frac{\operatorname{det}(w)}{\sqrt{\left|\operatorname{det}\left(p-w-w^{-1}\right)\right|}} \\
& \times \sum_{\sigma} \exp \left\{-r \pi i\left\langle\left(p-w-w^{-1}\right)^{-1} \sigma, \sigma\right\rangle\right\} \frac{\operatorname{vol} Z(w)}{|Z(G)|} .
\end{aligned}
$$

Here, the second sum is over $\sigma \in \Lambda^{R} /\left(p-w-w^{-1}\right) \Lambda^{R}$.

The TQFT result (4.16) in the canonical framing equals what we obtained (Proposition 5.15) from the semiclassical calculation, up to the factors vol $Z(w) /|Z(G)|$.

Remark 5.16. Exactness of the Semiclassical Approximation. Under a change of framing by one unit, the semiclassical formula for the $S U(2)$ Chern-Simons partition function varies like $\zeta$ rather than like $e^{2 \pi i c / 24}$. (This is predicted by the path integral [40], in which the framing enters the semiclassical approximation when one adds a "gravitational Chern-Simons" counterterm to remove the metric dependence.) Thus, in the natural framing $\psi(U)$ associated to the monodromy matrix $U$ for $\Sigma_{U}$, the semiclassical formula $Z_{\mathrm{sc}}\left(\Sigma_{U}, r ; \psi(U)\right)$ agrees precisely with $Z\left(\Sigma_{U}, r ; \psi(U)\right)$. There is a simple formal path integral argument for this result: see [18].

Remark 5.17. Unresolved Difficulties. For Witten invariants of mapping tori $\Sigma_{U}$, we have only been able to identify a large number of the factors appearing in the semiclassical approximation with factors appearing in the TQFT formulas. This is in contrast to the lens space case, where we have shown exact agreement between the TQFT formula and the semiclassical formula (in the limit $r \rightarrow \infty$ ). The ambiguities we have been unable to resolve, which prevent our identification from being complete, are the following:

1. The spectral flow factors need to be identified, as in Conjecture 5.8. Furthermore, Conjecture 5.8 should be rephrased to take account of points $A \in T \times T$ that are fixed by more than one $w U$. This might be done by introducing a family of operators interpolating between an operator corresponding to $U$ and an operator corresponding to $w U$, which would have spectral flow 2 if $\operatorname{det}(w)=-1$. Thus, if an element $A$ is fixed by both $U$ and $w U$, it would be natural for the corresponding terms in the 
semiclassical approximation to have different factors $(-i)^{I_{A}}$. The Maslov index (cf. [8]) may offer a natural interpretation.

2. For general groups $G$, the volumes of stabilizers $\operatorname{Stab}(\varrho) \cap Z(g)$ need to be computed.

3. In the case $G=S U(2)$, we have computed the volumes of stabilizers explicitly, and find that they give an appropriate normalization only in a metric that is different from the metric induced by the basic inner product. The latter metric however is the one giving the correct normalization for lens space Witten invariants.

4. We have not computed the integral of the torsion $\int_{\mathscr{B} \varrho} \tau^{1 / 2}$ when $\operatorname{Stab}(\varrho)$ is larger
than $T$.

Some of these ambiguities disappear if, instead of the semiclassical approximation to the Chern-Simons theory, one considers the semiclassical approximation to symplectic quantum mechanics: see [18].

\section{A. Appendix}

\section{A.1. Notation for Lie Groups}

We collect here our notation for various quantities associated to Lie groups, which will be used in this and the next chapter. The main reference for this material is the Appendix to [6]; see also [21].

1. We choose a compact Lie group $G$, which is assumed simple, connected and simply connected. It has maximal torus $T$ and Weyl group $W$. The usual alternating character on $W$ is denoted $w \rightarrow \operatorname{det}(w)$. The Lie algebras of $G$ and $T$ are respectively $\mathfrak{g}$ and $\mathfrak{t}$. The $\operatorname{rank}(\operatorname{dim} T)$ is denoted $l$.

2. We introduce the basic inner product $\langle$,$\rangle on \mathfrak{t}$, normalized so that the highest root has length $\sqrt{2}$.

3. "Tr" denotes a negative definite Ad-invariant quadratic form on $\mathfrak{g}$ : the normalization is such that on $\mathfrak{t}, \operatorname{Tr}(X Y)=-\langle X, Y\rangle$.

4. Roots will be denoted $\alpha$. Although roots are actually in the dual $\mathfrak{t}^{*}$, we use the basic inner product to identify them with elements of $t$. The set of positive roots will be denoted by $\Delta_{+}$. Simple roots will be denoted $\alpha_{i}, i=1, \ldots, l$.

5. The highest root is denoted $\alpha_{m}$.

6. Long and short roots: For any simple Lie algebra there are at most two possible lengths of roots, "long" and "short". The highest root is a long root, and has $\left\langle\alpha_{m}, \alpha_{m}\right\rangle=2$. Short roots then have $|\alpha|^{2}=2 / n$ for some $n \in \mathbb{Z}$. A simply laced Lie group is one for which all roots have the same length.

7. As usual, $\varrho$ will denote half the sum of the positive roots.

8. Coroots: To every root $\alpha \in \mathfrak{t}^{*}$ is associated a coroot $h_{\alpha} \in \mathfrak{t}$ such that $\alpha\left(h_{\alpha}\right)=2$. Using the basic inner product, this gives $h_{\alpha}=2 \alpha /\langle\alpha, \alpha\rangle$, and $\left|h_{\alpha}\right|^{2}=2 /\langle\alpha, \alpha\rangle$.

9. Lattices: We define two lattices in $t$. The coroot lattice $\Lambda^{R}$ is the lattice spanned by the coroots; because $G$ is simply connected, it equals the integer lattice. The weight lattice $\Lambda^{w}$ (which is dual to $\Lambda^{R}$ ) has a basis given by the fundamental weights $\lambda_{\alpha}$, which is the dual basis to the basis of coroots $h_{\alpha_{i}}$ for simple roots $\alpha_{i}$. Of course, since these lattices are dual, their volumes are inverses: vol $\Lambda^{w}=\left(\operatorname{vol} \Lambda^{R}\right)^{-1}$.

10. The fundamental Weyl chamber and its closure will be denoted FWC and $\overline{\text { FWC. }}$. 


\section{A.2. Notation Associated to Loop Group Representations}

We shall also need several quantities associated to loop groups, the definitions of which are rather more involved. $k \in \mathbb{Z}$ is the level, as before. This is in fact the same $k$ as appears multiplying the Chern-Simons Lagrangian in path integral formulation. 1. If $\lambda$ is a weight, then $Q_{\lambda}$, the quadratic Casimir of the corresponding representation, is

$$
Q_{\lambda}=\frac{1}{2}\left(|\lambda+\varrho|^{2}-|\varrho|^{2}\right) .
$$

2. The dual Coxeter number, denoted $h$, is the quadratic Casimir of the adjoint representation.

3. Freudenthal's strange formula

$$
\frac{|\varrho|^{2}}{2 h}=\frac{\operatorname{dim} G}{24}
$$

provides an expression for $h$.

4. The conformal weight of the representation indexed by the weight $\lambda$ is

$$
H_{\lambda}=\frac{Q_{\lambda}}{k+h} \text {. }
$$

5. The level $k$ central charge is

$$
c=\frac{(\operatorname{dim} G) k}{k+h}
$$

6. We denote $k+h$ by $r$.

\section{A.3. Theta Functions and Quantization for the Torus}

When $\Sigma$ is a torus, the vector space $\mathscr{H}_{\Sigma}^{(k)}$ arising from the quantization of the ChernSimons theory can be constructed quite explicitly. We define generating cycles $\alpha, \beta$ for $\pi_{1} \Sigma$. Then the moduli space of representations identifies with

$$
\mathscr{l} b=(T \times T) / W
$$

under $\varrho \rightarrow(\varrho(\alpha), \varrho(\beta))$. (Because the fundamental group is abelian, any representation must map the whole fundamental group into a maximal torus of $G$; then the only conjugation freedom left is the diagonal action of $W$.) The tangent space to $T \times T$ is

$$
\mathbb{A}=\mathfrak{t} \oplus \mathfrak{t}
$$

and

$$
T \times T=\mathbb{A} / \Lambda
$$

where

$$
\Lambda=\Lambda^{R} \oplus \Lambda^{R} .
$$

A choice of trivialization for a $G$ bundle $P \rightarrow \Sigma$ identifies $\mathbb{A}$ with a subspace of the space of connections $\mathscr{A}(\Sigma)$, and the product connection (denoted $A_{0}$ ) is identified with $0 \in \Sigma$. The semidirect product $W \tilde{\times} \Lambda$ identifies with the gauge transformations preserving $\mathbb{A}$ as a subspace of $\mathscr{b}$. The lattice in $\mathbb{A}$ is canonical, although the choice 
of $0 \in \mathbb{A}$ is not (gauge transformations may move 0 to another element of the lattice $\Lambda)$. The basic symplectic form $\omega$ on $\mathbb{A}$ is ([6], (5.19)):

$$
\begin{aligned}
\omega\left(\left(\xi_{1}, \eta_{1}\right),\left(\xi_{2}, \eta_{2}\right)\right) & =2 \pi\left(\left\langle\xi_{1}, \eta_{2}\right\rangle-\left\langle\xi_{2}, \eta_{1}\right\rangle\right) \\
& =-2 \pi\left\langle\left(\xi_{1}, \eta_{1}\right), S\left(\xi_{2}, \eta_{2}\right)\right\rangle
\end{aligned}
$$

for

$$
S=\left[\begin{array}{cc}
0 & -1 \\
1 & 0
\end{array}\right] \in S L(2, \mathbb{Z})
$$

We have:

Lemma A.1. If $(\mathbb{A}, \omega)$ is any symplectic affine space, then a connection $\theta$ on the trivial line bundle $\mathbb{A} \times \mathbb{C}$ with curvature $-i \omega$ is given by

$$
\theta_{A}(a)=-\frac{i}{2} \omega\left(A-A_{0}, a\right)
$$

for any $A_{0} \in \mathbb{A}$.

In our situation, we of course choose the reference connection $A_{0}$ to be the product connection, as above.

There is a line bundle $\mathscr{B}$ on $T \times T$, the prequantum line bundle, such that $c_{1}(\mathscr{B})=$ $\omega /(2 \pi)$. To construct it, we start with the trivial bundle $\mathbb{A} \times \mathbb{C} \rightarrow \mathbb{A}$ and quotient out by the action of $\Lambda$ lifted to $\mathbb{A} \times \mathbb{C}$ as follows ([6], 1(c):)

$$
(A, v) \sim\left(A+\lambda, e_{\lambda}(A) v\right)
$$

where

$$
e_{\lambda}(A)=\varepsilon(\lambda) \exp \left\{-(i / 2) \omega\left(A-A_{0}, \lambda\right)\right\}
$$

and the theta-characteristic $\varepsilon(\lambda) \in\{ \pm 1\}$ satisfies

$$
\varepsilon\left(\lambda_{1}+\lambda_{2}\right)=\varepsilon\left(\lambda_{1}\right) \varepsilon\left(\lambda_{2}\right)(-1)^{\omega\left(\lambda_{1}, \lambda_{2}\right) / 2 \pi} .
$$

To lift the action of $W$ to $\mathscr{L}$, one chooses the trivial lift from $\mathbb{A}$ to $\mathbb{A} \times \mathbb{C}$ :

$$
\tilde{\omega}(A, z)=\left(A_{0}+w\left(A-A_{0}\right), z\right) .
$$

This lift is equivariant with respect to the $\Lambda$ action, as we shall show in Lemma A.2. Both actions preserve the connection $\theta$.

Holomorphic sections of the line bundle $\mathscr{B}^{k}$ on $T \times T$ are given by theta functions. The following is treated in more detail in [6] (Sect. 5). A complex structure on $T \times T$ is defined by a modular parameter $\tau$ in the upper half plane $H$, which specifies a holomorphic structure on the torus $\Sigma$. For $u \in \mathfrak{t} \otimes \mathbb{C}$, we define

$$
\theta_{\gamma, k}(\tau, u)=\sum_{\alpha \in \Lambda^{R}} \exp \left\{i \pi k \tau\left|\alpha+\frac{\gamma}{k}\right|^{2}+2 \pi i k\left\langle u, \alpha+\frac{\gamma}{k}\right\rangle\right\} .
$$

Since we have lifted the action of $W$ to $\mathscr{L}, W$ also acts on $H^{0}(\mathscr{L})$. We may thus also define the Weyl anti-invariant linear combination

$$
\theta_{\gamma, k}^{-}=\sum_{w \in W} \operatorname{det}(w) \theta_{w(\gamma), k}
$$

The $\theta_{\gamma, k}$ are theta functions for $T \times T$ with the symplectic structure (A.7) and the complex structure given by $\tau$. 
One may define the level $k$ physical Hilbert space $\mathscr{H}_{\Sigma}^{(k)}$ as $H^{0}\left(\mathscr{L}^{k}, T \times T\right)^{W}$, the space of Weyl invariant holomorphic sections of $\mathscr{L}^{k}$. This is a vector space depending on the complex structure $\tau \in H$. The authors of [6] define a bundle over $H$ whose fibre over $\tau \in H$ is $\mathscr{H}_{\Sigma}^{(k)}(\tau)$, equipped with a projectively flat connection. The sections of the bundle over $H$ parallel under this connection turn out to be ([6], 5.44):

$$
\psi_{\gamma, k}(\tau, u)=\frac{\theta_{\gamma+\varrho, k+h}^{-}(\tau, u)}{\theta_{\varrho, h}^{-}(\tau, u)} .
$$

Here, a Weyl invariant theta function is obtained as the quotient of two Weyl antiinvariant theta functions. The quantity $\psi_{\gamma, k}$ (up to a phase) appears as the character for the level $k$ representation of the loop group $L G$ labelled by $\gamma$, in the Weyl-Kac character formula.

A basis for $\mathscr{H}_{\Sigma}^{(k)}$ is given by those level $k$ theta functions $\psi_{\lambda, k}$ corresponding to the highest weight integrable representations of the loop group $L G$ at level $k$ : i.e., by those labelled by weights $\lambda \in \mathfrak{t}$ such that for every positive root $\alpha$,

$$
k \geq\langle\lambda, \alpha\rangle \geq 0 \text {. }
$$

In fact, it is sufficient to demand that $\lambda$ be in the fundamental Weyl chamber and that its inner product with the highest root $\alpha_{m}$ be $\leq k$.

For the torus, $\mathscr{H}_{\Sigma}^{(k)}$ has an inner product in which the $\psi_{\gamma, k}$ form an orthonormal basis: this also provides an identification of $\mathscr{H}_{\Sigma}^{(k)}$ with its dual. The vector in $\mathscr{H}_{\Sigma}^{(k)}$ corresponding to the solid torus under the axioms of topological field theory is $\psi_{0, k}$, the vector labelled by weight 0 . The other $\psi_{\lambda, k}$ correspond to the solid torus with a longitudinal Wilson line in the representation $\lambda$.

\section{A.4. Liftings to the Prequantum Line Bundle}

This subsection proves the following technical lemma:

Lemma A.2. For a suitable choice of the theta-characteristic $\varepsilon$, the lift $\tilde{f}_{U}$ of $f_{U}: T \times$ $T \rightarrow T \times T$ corresponding to $U \in S L(2, \mathbb{Z})$ given by $(5.12)$ and the lift of $w \in W$ given in Sect.A.3 are equivariant with respect to the action of the lattice $\Lambda$ on the line bundle $\mathscr{L} \rightarrow T \times T$, which is defined in (A.9).

Proof. Write $V$ for the linear maps on $\mathfrak{t} \oplus \mathfrak{t}$ corresponding to $U \in S L(2, \mathbb{Z})$ and $w \in W$. The equivariance condition is characterized by the following equation on $\mathscr{B}=\mathbb{A} \times \mathbb{C}:$

$$
\left(V A+V \lambda, e_{\lambda}(A) v\right)=\left(V A+V \lambda, e_{V(\lambda)}(V A) v\right)
$$

Now from (A.9) we have

$$
\frac{e_{V(\lambda)}(V A)}{e_{\lambda}(A)}=\frac{\varepsilon(V \lambda)}{\varepsilon(\lambda)},
$$

so we need $\varepsilon(V \lambda)=\varepsilon(\lambda)$. Actually we need only check this for $\lambda$ in some basis of lattice vectors.

We fix the coroot basis $\left\{h_{\alpha}\right\}$ of $\Lambda^{R}$ (see Sect. A.1) and correspondingly a basis $\left\{h_{\alpha}^{(1)}, h_{\alpha}^{(2)}\right\}$ of $\Lambda=\Lambda^{R} \oplus \Lambda^{R}$. We define the theta-characteristic by

$$
\varepsilon\left(h_{\alpha}^{(i)}\right)=1
$$


Then for $U \in S L(2, \mathbb{Z}), U h_{\alpha}^{(i)}=m h_{\alpha}^{(1)}+n h_{\alpha}^{(2)}$ for some $m, n \in \mathbb{Z}$; because $h_{\alpha}$ does not mix with the other coroots $h_{\beta}, \beta \neq \alpha$ under $U$ and because $\left\langle h_{\alpha}, h_{\alpha}\right\rangle \in 2 \mathbb{Z}$, we have $\varepsilon\left(U h_{\alpha}^{(i)}\right)=1$.

Similarly for $w \in W, w h_{\alpha}^{(i)}=\sum_{\beta} n_{\beta} h_{\beta}^{(i)}$ : the two summands $\mathfrak{t}_{1}$ and $\mathfrak{t}_{2}$ in $\mathfrak{t} \oplus \mathfrak{t}$ do not mix. So since $\omega$ pairs $\mathfrak{t}_{1}$ with $\mathfrak{t}_{2}$, again $\varepsilon\left(w h_{\alpha}^{(i)}\right)=1$. Hence the Definition (5.12) does indeed give a $\Lambda$-equivariant lift to $\mathscr{L}$.

Remark A.3: Theta-Characteristics. The choice of a theta characteristic for a bundle $\mathscr{B}$ on $T \times T$ is the specification of $w_{1}(\mathscr{L}) \in H^{1}\left(T \times T, \mathbb{Z}_{2}\right)$. We know that $(T \times T) / W$ is simply connected, so bundles with different choices of theta-characteristic on $T \times T$ descend to isomorphic bundles on $(T \times T) / W$, and the choice of theta-characteristic is irrelevant for our purposes. For convenience in specifying the lift of $S L(2, \mathbb{Z})$ to $\mathscr{L}$, we make the particular choice (A.17) for the theta characteristic. A different choice would force us to choose a different lift in order that the lift be equivariant with respect to the $\Lambda$ action.

The theta characteristic we have chosen is obviously identically 1 in the $S U(2)$ case.

\section{A.5. Alcoves}

Suppose $G$ is a simply laced Lie group, i.e., all the roots have the same length.

1. The affine Weyl group $W_{\text {aff }}$ is the semidirect product of the ordinary Weyl group $W$ and the translations given by the coroot lattice $\Lambda^{R}$. It has an obvious action on $t$. 2. An alcove is a fundamental domain of $W_{\text {aff }}$. One may define a distinguished alcove, the fundamental alcove $C_{0}$ in $\mathfrak{t}$, by

$$
C_{0}=\left\{x \in \overline{\mathrm{FWC}} \mid\left\langle x, \alpha_{m}\right\rangle \leq 1\right\},
$$

where $\alpha_{m}$ is the highest root and FWC the fundamental Weyl chamber.

3. A basic cell of $\Lambda^{R}$ thus contains $|W|$ alcoves. Hence also a basic cell of $\Lambda^{w}$ contains $|W| \operatorname{vol}\left(\Lambda^{w}\right) / \operatorname{vol}\left(\Lambda^{R}\right)$ alcoves.

4. By extension, we may define $k$-alcoves as fundamental domains of the action of the semidirect product $W \tilde{x} k \Lambda^{R}$. The set of weights indexing the level $k$ integrable representations of the loop group (A.16) are then the weights in the fundamental $k$-alcove

$$
C_{0}(k)=\left\{x \in \overline{\mathrm{FWC}} \mid\left\langle x, \alpha_{m}\right\rangle \leq k\right\} .
$$

5. Using the formula (A.1) for $h$, it follows that

$$
\left\langle\alpha_{m}, \varrho\right\rangle=h-1 \text {. }
$$

Thus translating the set of weights in the fundamental $k$-alcove by $\varrho$ transforms them into the set of weights in the interior of the fundamental $(k+k)$-alcove.

\section{References}

1. Atiyah, M.F.: The logarithm of the Dedekind $\eta$ function. Math. Ann. 278, 335-380 (1987)

2. Atiyah, M.F.: On framings of 3-manifolds. Topology 29, 1-8 (1990)

3. Atiyah, M.F.: The Geometry and Physics of Knots. Cambridge: Cambridge Univ. Press (1990) 
4. Atiyah,' M.F., Patodi, V., Singer, I.M.: Spectral asymmetry and Riemannian geometry I, II, III. Math. Proc. Camb. Phil. Soc. 77, 43-69 (1975); 78, 405-432 (1975); 79, 71-99 (1976)

5. Axelrod, S.: Geometric Quantization of Chern-Simons Gauge Theory, Ph.D. Thesis, Princeton University 1991

6. Axelrod, S., Della Pietra, S., Witten, E.: Geometric quantization of Chern-Simons gauge theory. J. Diff. Geo. 33, 787-902 (1991)

7. Chandrasekharan, K.: Elliptic Functions (Grundlehren der math. Wissenschaften, vol. 281). Berlin Heidelberg New York 1985

8. Dostoglou, S., Salamon, D.: Instanton homology and symplectic fixed points. Warwick preprint (1990)

9. Elitzur, S., Moore, G., Schwimmer, A., Seiberg, N.: Remarks on the canonical quantization of the Chern-Simons-Witten theory. Nucl. Phys. B 326, 108-134 (1989)

10. Franz, W.: Über die Torsion einer Überdeckung. J. Reine Angew. Mathematik 173. 245-254 (1935)

12. Freed, D.: Reidemeister torsion, spectral sequences and Brieskorn spheres. Austin preprint (1990)

13. Garoufalidis, S.: Relations among 3-manifold invariants. University of Chicago preprint (1991)

14. Gepner, D., Witten, E.: String theory on group manifolds. Nucl. Phys. B 278, 493-549 (1986)

15. Hirzebruch, F.: The signature theorem: Reminiscences and recreation. In: Prospects in Mathematics (Ann. Math. Studies 70) pp. 3-31. Princeton, NJ: Princeton Univ. Press (1971)

16. Hirzebruch, F., Neumann, W., Koh, H.: Differentiable Manifolds and Quadratic Forms. New York: Marcel Dekker 1971

17. Hirzebruch, F., Zagier, D.: The Atiyah-Singer Index Theorem and Elementary Number Theory. Publish or Perish 1974

18. Jeffrey, L.C.: Symplectic quantum mechanics and Chern-Simons gauge theory (in preparation)

19. Jeffrey, L.C., Weitsman, J.: Half-Density Quantization of the Moduli Space of Flat Connections and Witten's Semiclassical Manifold Invariants, preprint IASSNS-HEP-91/94

20. Johnson, D.: A geometric form of Casson's invariant and its connection to Reidemeister Torsion, UCLA lecture notes (1988)

21. Kac, V.: Infinite Dimensional Lie Algebras (Progress in Mathematics, vol. 44). Basel: Birkhäuser 1983

22. Kirby, R., Melvin, P., Evaluations of the three-manifold invariants of Witten and ReshetikhinTuraev for $\mathfrak{s l}(2, \mathbb{C})$. In: Donaldson, S.K., Thomas C.B. (eds.). Proc. of LMS Symposium on the Geometry of Low Dimensional Manifolds (Durham, 1989) LMS Mathematics Lecture Series vol. 150

23. Kirby, R., Melvin, P.: The three-manifold invariants of Witten and Reshetikhin-Turaev. Invent. Math. 105, 473-545 (1991)

24. Kirby, R., Melvin, P.: Dedekind sums, mu-invariants and the signature cocycle, preprint (1992)

25. Kirby, R., Melvin, P.: In preparation

26. Kirk, P., Klassen, K.: Chern-Simons invariants of 3-manifolds and representation spaces of knot groups. Math. Ann. 287, 343-367 (1990)

27. Kohno, T., Topological invariants for 3-manifolds using representations of mapping class groups I, Kyushu Univ. Preprint (1990)

28. Lang, S.: Algebraic Number Theory. New York: Addison-Wesley 1970

29. Majid, S., Soibelman, Ya.S.: Rank of quantum universal enveloping algebras and modular functions. Commun. Math. Phys. 137, 249-262 (1991)

30. Moore, G., Seiberg, N.: Classical and quantum conformal field theory. Commun. Math. Phys. 123, 77 (1989)

31. Mumford, D.: Tata Lectures on Theta I. Progress in Mathematics, vol.28. Basel: Birkhäuser 1982

32. Pressley, A., Segal, G.: Loop Groups. Oxford: Oxford University Press 1988

33. Rademacher, H.: Topics in Analytic Number Theory. Grundlehren der math. Wissenschaften, vol. 169). Berlin Heidelberg New York: Springer 1973

34. Ramadas, T.R., Singer, I.M., Weitsman, J.: Some comments on Chern-Simons gauge theory. Commun. Math. Phys. 126, 409-430 (1989)

35. Ray, D., Singer, I.M.: R-torsion and the Laplacian on Riemannian manifolds. Adv. Math. 7, $145-210(1971)$ 
36. Reshetikhin, N., Turaev, V.: Ribbon graphs and their invariants derived from quantum groups. Commun. Math. Phys. 127, 1-26 (1990); Invariants of three-manifolds via link polynomials and quantum groups. Invent. Math. 103, 547-597 (1991)

37. Rolfsen, D.: Knots and Links. Publish or Perish 1976

38. Skoruppa, N., Zagier, D.: A trace formula for Jacobi forms. J. Reine. Angew. Math. 393, 168-198 (1989)

39. Walker, K.: On Witten's three-manifold invariants, preprint (1991)

40. Witten, E.: Quantum field theory and the Jones polynomial. Commun. Math. Phys. 121, 351-399 (1989)

41. Witten, E.: On quantum gauge theories in two dimensions, IAS preprint IASSNS-HEP-91/3 (1991)

Communicated by A. Jaffe 